\title{
Stealth decaying spin-1 dark matter
}

\author{
Cédric Delaunay, ${ }^{a}$ Teng $\mathrm{Ma}^{b}$ and Yotam Soreq ${ }^{b}$ \\ ${ }^{a} L A P T h, C N R S-U S M B$, \\ BP 110 Annecy-le-Vieux, F-74941 Annecy, France \\ ${ }^{b}$ Physics Department, Technion - Israel Institute of Technology, \\ Haifa 3200003, Israel \\ E-mail: cedric.delaunay@lapth.cnrs.fr, t.ma@campus.technion.ac.il, \\ soreqy@physics.technion.ac.il
}

ABSTRACT: We consider models of decaying spin-1 dark matter whose dominant coupling to the standard model sector is through a dark-Higgs Yukawa portal connecting a TeVscale vector-like lepton to the standard model (right-handed) electron. Below the electronpositron threshold, dark matter has very slow, loop-suppressed decays to photons and (electron) neutrinos, and is stable on cosmological time-scale for sufficiently small gauge coupling values. Its relic abundance is set by in-equilibrium dark lepton decays, through the freeze-in mechanism. We show that this model accommodates the observed dark matter abundance for natural values of its parameters and a dark matter mass in the $\sim 5 \mathrm{keV}$ to $1 \mathrm{MeV}$ range, while evading constraints from direct detection, indirect detection, stellar cooling and cosmology. We also consider the possibility of a nonzero gauge kinetic mixing with the standard model hypercharge field, which is found to yield a mild impact on the model's phenomenology.

Keywords: Phenomenological Models

ArXiv EPrint: 2009.03060 


\section{Contents}

1 Introduction 1

2 Light dark matter from a feebly gauged dark $\mathrm{U}(1)_{X}$ group 3

3 The VDM relic density $\quad 7$

$\begin{array}{lll}3.1 & \text { Freeze-in from heavy lepton decay } & 7\end{array}$

3.2 Subleading scattering contributions 9

$\begin{array}{ll}3.3 & \text { The relativistic freeze-out limit } \\ \end{array}$

$4 \quad$ DM phenomenology and collider signatures $\quad 13$

4.1 Direct detection 13

$\begin{array}{lll}4.2 & \text { Indirect detection } & 15\end{array}$

$\begin{array}{lll}4.3 & \text { Astrophysical and cosmological constraints } & 15\end{array}$

$\begin{array}{ll}4.4 \text { Collider signatures } & 19\end{array}$

5 Conclusions 20

\section{Introduction}

Dark matter (DM) is perhaps the most fascinating puzzle in high energy physics today. The existence of DM is supported by different observations from galactic to cosmic scales. However very little is known about its nature. For instance, it is not even clear that DM is made of fundamental particles. If so, most properties of particle DM, like its mass, spin and relic abundance generation mechanism, are unknown. Moreover, the observational evidence for DM is all based on gravity, and the existence of other types of interaction with the Standard Model (SM) fields remain to be discovered.

Non-gravitational interactions of the DM are often advocated to explain the production of its relic abundance in the early universe. This assumption triggered hope to directly detect DM in terrestrial experiments [1] or to produce it at colliders. Despite a sustained effort over several decades, experiment has failed to discover theoretically well-motivated DM candidates, such as weakly interacting massive particles (WIMPs) [2-4] and axion DM $[5,6]$. This null result calls for either alternative candidates [7] or alternative relic production mechanisms, and perhaps even questions the very existence of DM interactions beyond gravity [8-11].

One possible alternative scenario consists in realizing the DM as feebly interacting massive particles (FIMPs) that are produced through the freeze-in mechanism $[12,13]$. See ref. [14] for a recent review. Unlike the standard thermal scenario where the DM is in equilibrium with the SM until its annihilation rate is beaten by Hubble expansion leading 
to DM freeze-out, within the freeze-in scenario the DM is never in thermal equilibrium with the SM and is gradually produced from scattering or decay of SM particles. Despite its non thermal nature the freeze-in mechanism, similarly to the freeze-out one, is typically not sensitive to initial conditions in the very early Universe, with the known exception of UVdominated freeze-in [15]. Consequently, the relic abundance only depends on DM properties that can be probed in experiments. Also, FIMP may be related to leptogenesis [16].

Massive spin-1 particles are interesting DM candidates. In particular, for masses less than that of the electron-positron pair, new vectors can only decay to $3 \gamma$ or $\nu \bar{\nu}$ with typically highly suppressed decay widths, such that vector DM (VDM) particles are easily stable on cosmological time scales without resorting to an ad-hoc stabilizing symmetry. A similar feature is observed in models of scalar DM decaying to SM fields through the Higgs portal $[17,18]$. A well-know VDM candidate enjoying this property is dark photon DM [1921], which only couples to the SM through kinetic mixing with the hypercharge field [22-24]. However, it is rather challenging to construct a viable model of dark photon DM below the $\mathrm{MeV}$ scale. For instance, a thermally produced relic abundance is excluded by direct detection searches $[20,25,26]$ and in strong tension with stellar cooling [27-29] and cosmological bounds [30]. Sufficient dark photon production is still achievable nonthermally. For instance, dark photons can be produced during inflation by a misalignment mechanism [31] or quantum fluctuations [31, 32]. Another possibility is dark photon production from an oscillating axion field [33-36]. Finally, nonthermally produced massive spin-1 DM may also be related to neutrino masses and leptogenesis in the context of $B-L$ models [37].

In this work we explore a model of sub-MeV VDM resulting from the spontaneous breakdown of a very weakly gauged dark $\mathrm{U}(1)_{X}$ group. Its relic abundance is created from the decay of a heavy dark fermion in thermal equilibrium with the SM. The dark fermion is a massive isospin singlet vector-like lepton at the $\mathrm{TeV}$ scale or higher, unit dark charge and hypercharge -1 , which decays to VDM thanks to mass mixing with the SM leptons. There are two possible VDM production regimes, depending on the strength of the dark sector interactions with the SM. For feeble coupling values the VDM relic density is set by the freeze-in mechanism, whereas in the limit of couplings large enough to bring VDM in equilibrium with the thermal bath, VDM freezes out while being still relativistic once the dark fermion decouples. The latter case is reminiscent of the forbidden DM scenario [38], although in a limit where DM is much lighter than the mediator.

This minimal setup has several interesting features. First of all, there is a parametric suppression of the VDM coupling to SM lepton pairs relative to its coupling to one SM and one dark lepton. This allows to saturate the observed DM relic abundance while evading all existing constraints. Then, the VDM-to-SM couplings needed for DM production are all technically natural, and can thus assume arbitralily small values without introducing finetuning. As a result, there is a large portion of parameter space where DM can be naturally produced to the observed level and yet remain invisible to all DM detection experiments. Finally, in the relativistic freeze-out limit, the comoving VDM number density at freeze-out is independent of its mass and couplings, up to possible change of the number of entropy degrees of freedom during freeze-out. Hence, in this regime, the observed DM abundance requires a hot $\mathrm{DM}$ with $\mathrm{a} \sim 60 \mathrm{eV}$ mass, which is in tension with large-scale structure (LSS) formation [39-41]. 
The rest of the paper is organized as follows. We define our VDM model in section 2, calculate its relic abundance in section 3 and present its DM-related phenomenology and collider signatures in section 4 . We summarize our conclusions in section 5 .

\section{Light dark matter from a feebly gauged dark $\mathrm{U}(1)_{X}$ group}

In this section, we present a minimal model of VDM with a sub-MeV mass. Consider, in addition to the SM symmetries and fields, a $U(1)_{X}$ gauge group with a tiny gauge coupling $g_{X} \ll 1$ which is spontaneously broken by a dark Higgs $\phi$ of $\mathrm{U}(1)_{X}$ charge unity. Consider also a dark vector-like fermion $E$ which has the same charge as $\phi$ under $\mathrm{U}(1)_{X}$ and carries hypercharge -1 . We assume all SM fields to be $\mathrm{U}(1)_{X}$ neutral.

The most general renormalizable Lagrangian is then,

$$
\mathcal{L}=\mathcal{L}_{\mathrm{SM}}+\mathcal{L}_{\text {dark }}+\mathcal{L}_{\text {portal }}
$$

where $\mathcal{L}_{\mathrm{SM}}$ is the SM Lagrangian and

$$
\begin{aligned}
\mathcal{L}_{\text {dark }} & =-\frac{1}{4} \chi_{\mu \nu} \chi^{\mu \nu}+\bar{E}\left(i \not D-M_{E}\right) E+\left(D_{\mu} \phi\right)^{\dagger} D^{\mu} \phi-V(\phi), \\
\mathcal{L}_{\text {portal }} & =-\lambda_{\phi H}|\phi|^{2}|H|^{2}-\frac{\epsilon}{2} \chi_{\mu \nu} B^{\mu \nu}-\left(x_{E} \bar{E}_{L} e_{R} \phi+\text { h.c. }\right)
\end{aligned}
$$

where $H$ is the SM-Higgs field, $B_{\mu}$ is the hypercharge gauge field, with gauge coupling $g_{Y}$, and $e_{R}$ denotes the right-handed (RH) SM leptons.

We assume that $V(\phi)=\lambda_{\phi}\left(|\phi|^{2}-w^{2} / 2\right)^{2}$ is unstable at the origin and has its global minimum at $w>0$. In unitary gauge $\phi=(\varphi+w) / \sqrt{2}$ where $\varphi$ denotes a real scalar fluctuation around the non zero vacuum expectation value (VEV) $w$. This spontaneous breakdown induces a mass for the $\mathrm{U}(1)_{X}$ gauge field of $m_{\chi}=g_{X} w$. Note that a natural value of the dark Higgs VEV is (assuming the quartic coupling dominates the quantum correction to the $\phi$ mass squared) $w \sim \lambda_{\phi}^{1 / 2} \Lambda / 4 \pi$ where $\Lambda$ is the cutoff scale of the model. Hence, a moderately large cutoff of, for instance, $10^{5} \mathrm{TeV}$ together with a vector mass $m_{\chi}<2 m_{e}$ implies a tiny value of the $\mathrm{U}(1)_{X}$ gauge coupling of

$$
g_{X} \sim 10^{-13} \frac{m_{\chi \mathrm{keV}}}{\lambda_{\phi}^{1 / 2}}\left(\frac{10^{5} \mathrm{TeV}}{\Lambda}\right),
$$

where $m_{\chi \mathrm{keV}}$ is the VDM mass in units of $\mathrm{keV}$. For fixed $m_{\chi}$ and $\lambda_{\phi}$, larger values of $g_{X}$ are possible at the expense of fine-tuning the VEV $w$ below its natural value (or lowering the cutoff scale), while smaller values can be obtained by raising the scale $\Lambda$. Another implication of naturalness is that the scalar fluctuation $\varphi$ is very heavy $m_{\varphi} \sim \lambda_{\phi}^{1 / 2} \Lambda / 4 \pi \sim$ $8 \times 10^{4} \lambda_{\phi}^{1 / 2}\left(\Lambda / 10^{5} \mathrm{TeV}\right) \mathrm{TeV}$, unless the quartic coupling is very small. Henceforth, we assume $\lambda_{\phi} \sim \mathcal{O}(1)$. The vector-like fermion mass $M_{E}$ can be anywhere between a few hundred $\mathrm{GeV}$ and $\Lambda$, lighter masses being in tension with collider constraints. In the subsequent sections, we will focus on $M_{E}$ in the TeV range for definiteness.

The operators in $\mathcal{L}_{\text {portal }}$ are portal interactions linking the dark sector states to the SM. The first two terms are the Higgs and kinetic mixing portals, respectively, while the 
last one is a leptonic Yukawa portal. While the Higgs portal yields a rich phenomenology when the scalar $\varphi$ is light, see e.g. ref. [42] for a review, in our case the scalar fluctuation is close to the cutoff scale and totally decoupled. Therefore, for simplicity, we set $\lambda_{\phi H}=0$.

The kinetic mixing however is not always negligible. Even if set to zero at the classical level, it is generated at the one-loop level from states carrying both hypercharge and $\mathrm{U}(1)_{X}$ charge. There is only one such fermion in our model, giving [24]

$$
\epsilon_{\mathrm{loop}}=\frac{g_{X} g_{Y}}{6 \pi^{2}} \log \left(\frac{M_{E}}{\Lambda}\right)
$$

which is logarithmically divergent and thus sensitive to an unknown UV contribution. The loop contribution to the kinetic mixing could be made calculable by adding extra fields. For example, consider an additional heavy vector-like fermion $E^{\prime}$ of mass $M_{E}^{\prime}$, hypercharge -1 and $\mathrm{U}(1)_{X}$ charge opposite to that of $E$. Then, the total one-loop contribution is finite with $\epsilon_{\text {loop }}=g_{X} g_{Y} /\left(6 \pi^{2}\right) \log \left(M_{E} / M_{E^{\prime}}\right)$. The kinetic mixing even vanishes in the limit of degenerate fermions $\left(M_{E}=M_{E^{\prime}}\right)$, which may result from an approximate $Z_{2}$ symmetry. In principle, this would require to include an additional portal operator like $-x_{E^{\prime}} \overline{E^{\prime}}{ }_{L} e_{R} \phi^{\dagger}+$ h.c. which would not change the phenomenology. Therefore, in this case one could set $x_{E^{\prime}}=0$ for simplicity, so the only effect of $E^{\prime}$ would be to regulate the kinetic mixing. ${ }^{1}$ To summarize $\epsilon$ is essentially a free parameter of the model, whose precise value depends on UV physics. In order to get a feeling of this freedom on the model's phenomenology, we investigate two representative cases in the following, where $\epsilon=0$ and $\epsilon=g_{X} g_{Y} /\left(6 \pi^{2}\right)$.

After $\mathrm{U}(1)_{X}$ breaking, $E$ mixes with the SM electron. The corresponding mass matrix is diagonalized by the mixings angles

$$
\tan \left(2 \theta_{R}\right)=\frac{2 \sqrt{2} M_{E} x_{E} w}{2 M_{E}^{2}-\left(y_{e} v\right)^{2}-\left(x_{E} w\right)^{2}},
$$

between $e_{R}$ and $E_{R}$, and

$$
\tan \left(2 \theta_{L}\right)=\frac{2 y_{e} v x_{E} w}{2 M_{E}^{2}-\left(y_{e} v\right)^{2}+\left(x_{E} w\right)^{2}}
$$

between $e_{L}$ and $E_{L}$, where $v \approx 246 \mathrm{GeV}$ is the SM Higgs VEV and $y_{e}$ is the electron Yukawa. Its eigenvalues, denoted $m_{e}$ and $m_{E}$ (with $m_{e}<m_{E}$ ), obey

$$
m_{e} m_{E}=y_{e} v M_{E} / \sqrt{2}, \quad m_{e}^{2}+m_{E}^{2}=M_{E}^{2}+\left[\left(y_{e} v\right)^{2}+\left(x_{E} w\right)^{2}\right] / 2,
$$

where $m_{e} \approx 511 \mathrm{keV}$ is identified with the physical electron mass. In the limit of $M_{E} \gg$ $y_{e} v$ and $x_{E} w$ that we envisage here, we have approximately $\theta_{R} \approx x_{E} w /\left(\sqrt{2} M_{E}\right)$ and $\theta_{L} \approx y_{e} v \theta_{R} /\left(\sqrt{2} M_{E}\right) \ll \theta_{R}$ and the physical masses are corrected at $\mathcal{O}\left(\theta_{R}^{2}\right)$ relative to the unmixed case as $m_{e} \approx y_{e} v / \sqrt{2}\left(1-\theta_{R}^{2} / 2\right)$ and $m_{E} \approx M_{E}\left(1+\theta_{R}^{2} / 2\right)$.

\footnotetext{
${ }^{1}$ This assumption explicitly breaks the $Z_{2}$ symmetry and radiatively lifts the mass degeneracy, thus, reintroducing a nonzero $\epsilon_{\text {loop. }}$. However, we will consider a regime where $x_{E} \ll 1$ so that this breaking is small and negligible in practice.
} 
The mass mixing allows the dark vector $\chi_{\mu}$ to interact with the electron through the Lagrangian ( $e$ and $E$ now denoting mass eigenstates)

$$
\begin{aligned}
\mathcal{L}_{\mathrm{int}} & =j_{X}^{\mu} \chi_{\mu} \\
j_{X}^{\mu} & =\bar{e} \gamma^{\mu}\left(g_{\chi e e}^{V}+g_{\chi e e}^{A} \gamma_{5}\right) e+\bar{E} \gamma^{\mu}\left(g_{\chi E E}^{V}+g_{\chi E E}^{A} \gamma_{5}\right) E+\left[\bar{E} \gamma^{\mu}\left(g_{\chi E e}^{V}+g_{\chi E e}^{A} \gamma_{5}\right) e+\text { h.c. }\right],
\end{aligned}
$$

where $g^{V, A} \equiv\left(g^{R} \pm g^{L}\right) / 2$ and (at linear order in $\epsilon$, neglecting the subleading mixing contribution with the $Z[43,44])$

$$
g_{\chi e e}^{L, R} \approx g_{X} s_{L, R}^{2}+\epsilon e c_{W}, \quad g_{\chi E e}^{L, R} \approx g_{X} c_{L, R} s_{L, R}, \quad g_{\chi E E}^{L, R} \approx g_{X} c_{L, R}^{2}+\epsilon e c_{W},
$$

where $c_{W}\left(s_{W}\right) \equiv \cos \theta_{W}\left(\sin \theta_{W}\right), \theta_{W}$ is the weak mixing angle and $c_{L, R} \equiv \cos \theta_{L, R}, s_{L, R} \equiv$ $\sin \theta_{L, R}$. In the absence of kinetic mixing, the LH couplings are suppressed by a factor of $\mathcal{O}\left(m_{e} / m_{E}\right)$ relative to the $\mathrm{RH}$ ones and $g_{\chi e e}^{R} / g_{\chi E e}^{R} \approx \theta_{R} \ll 1$. This implies in particular that VDM production from annihilations of electron pairs will be parametrically suppressed, and typically negligible, compared to that from in-equilibrium $E$ decays which is controlled by the $E \rightarrow \chi e$ partial width given by

$$
\Gamma_{E \rightarrow \chi e} \simeq \frac{g_{X}^{2} \theta_{R}^{2} m_{E}^{3}}{32 \pi m_{\chi}^{2}} \simeq \frac{x_{E}^{2} m_{E}}{64 \pi},
$$

to leading order in $\theta_{R}$. Note that the enhancement factor $\left(m_{E} / m_{\chi}\right)^{2}$ arising from the longitudinal polarization of $\chi_{\mu}$ cancels out with a similar factor from the mixing angle, signaling that $E$ decays actually to the Goldstone boson of the broken $\mathrm{U}(1)_{X}$ symmetry through the Yukawa portal operator. Electroweak (EW) decays $E \rightarrow Z e$ and $E \rightarrow W \nu$ are also possible, with the following widths (again, to leading order in $\theta_{R}$ )

$$
\Gamma_{E \rightarrow Z e} \simeq \frac{g_{Z}^{2} \theta_{R}^{2} m_{E} m_{e}^{2}}{128 \pi m_{Z}^{2}}\left(1+4 \epsilon^{2} \frac{g_{X}^{2} m_{E}^{2}}{g_{Z}^{2} m_{e}^{2}}\right), \quad \Gamma_{E \rightarrow W \nu} \simeq \frac{g^{2} \theta_{R}^{2} m_{E} m_{e}^{2}}{64 \pi m_{W}^{2}},
$$

where $g$ is the $\mathrm{SU}(2)_{L}$ gauge coupling, $g_{Z} \equiv g / c_{W}$, and assuming the large $m_{E}$ limit. Note that, in the absence of kinetic mixing, the decay to $Z e$ is mediated only by the LH mixing angle $\theta_{L} \simeq m_{e} \theta_{R} / m_{E}$.

The dark vector, $\chi_{\mu}$, is not stabilized by any symmetry and decays. For $m_{\chi}<2 m_{e}$ the leading decay channels are in three-photons, the two-photon decay being forbidden by the Yang theorem [45], and $\nu \bar{\nu}$ final states. In the Euler-Heisenberg effective Lagrangian limit $[46]$ the $3 \gamma$ decay rate is $[19,20]$

$$
\Gamma_{\chi \rightarrow 3 \gamma} \simeq \frac{17 \alpha^{3}}{360^{3} \pi^{4}} \frac{m_{\chi}^{9}}{m_{e}^{8}}\left[g_{X}\left(\frac{\theta_{R}^{2}}{2}+\frac{m_{e}^{4}}{m_{E}^{4}}\right)+\epsilon e c_{W}\right]^{2},
$$

where the large $m_{E}$ limit was assumed and sub-leading terms of $\mathcal{O}\left(\epsilon m_{e}^{4} / m_{E}^{4}\right)$ and $\mathcal{O}\left(\theta_{R}^{2} m_{e}^{2} / m_{E}^{2}\right)$ have been neglected. The $m_{e}^{4} / m_{E}^{4}$ term denotes the $E$ loop contribution to the decay amplitude. While the effective field theory treatment of the $3 \gamma$ decay is always valid for $E$ particles running in the loop, the electron contribution in eq. (2.14) receives large corrections beyond the effective result for $100 \mathrm{keV} \lesssim m_{\chi}<2 m_{e}$ [47], which 

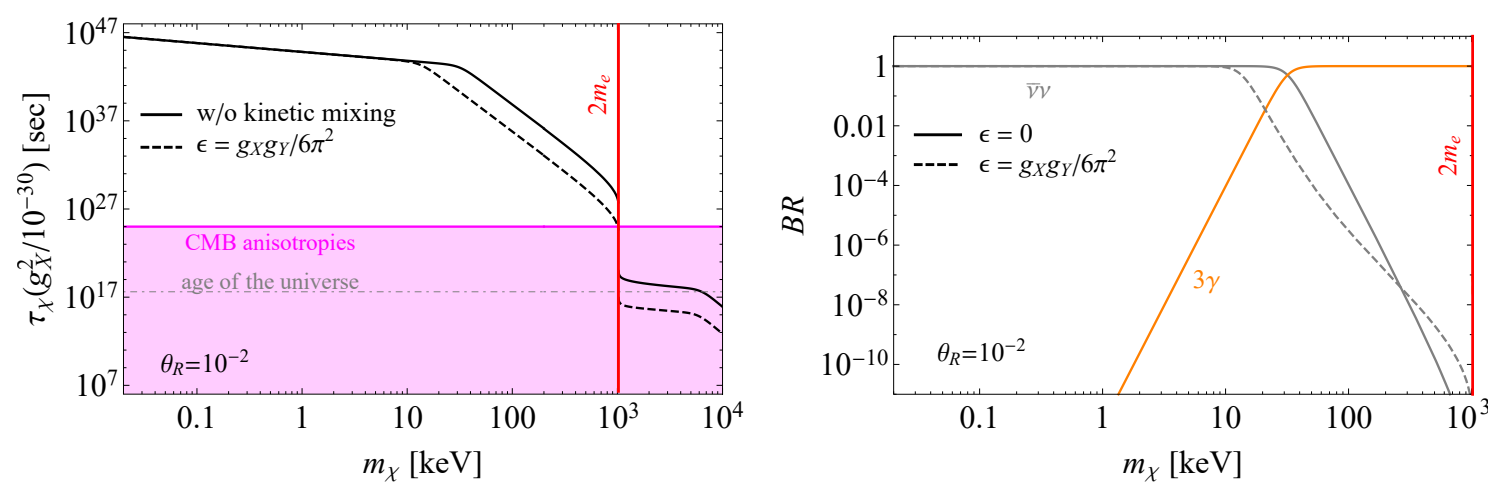

Figure 1. Lifetime (left) and BRs (right) of decaying VDM as function of the VDM mass $m_{\chi}$, for $m_{E}=1 \mathrm{TeV}$ and $\theta_{R}=10^{-2}$. Solid (dashed) lines assumes zero kinetic mixing $\left(\epsilon=g_{X} g_{Y} /\left(6 \pi^{2}\right)\right)$. Note that $\tau_{\chi} \propto g_{X}^{-2}$ and that all BRs are independent of $g_{X}$, as well as $\theta_{R}$ in the absence of kinetic mixing. The dot-dashed line denotes the present age of the Universe and the shaded magenta region is excluded by studies of CMB anisotropies. The vertical red line denotes the electron-positron threshold $m_{\chi}=2 m_{e}$.

we include in our numerical evaluation of $\Gamma_{\chi \rightarrow 3 \gamma}$. The decay rate into neutrinos is induced from $W$ loops and from tree-level $\chi-Z$ mass mixing for non-vanishing kinetic mixing, which gives (keeping only the dominant logarithmic part at one-loop)

$$
\Gamma_{\chi \rightarrow \nu \bar{\nu}} \simeq \frac{m_{\chi}}{24 \pi}\left[\theta_{R}^{2} \frac{g_{X} g^{2}}{64 \pi^{2}} \frac{m_{e}^{2}}{m_{W}^{2}} \log \left(\frac{m_{E}^{2} m_{W}^{6}}{m_{e}^{8}}\right)+\epsilon g_{Y} \frac{m_{\chi}^{2}}{2 m_{Z}^{2}}\right]^{2} .
$$

The first term in the bracket assumes the large $m_{E}$ limit, while the second term is the tree-level contribution from mixing with the $Z$ boson. Note that the kinetic mixing does not contribute at loop-level in the $m_{\chi} \rightarrow 0$ limit, since any $\mathrm{U}(1)_{X}$ operator coupling the $\chi_{\mu}$ field to the LH neutrino current must involve the dark Higgs field. For $m_{\chi}>2 m_{e}$, the dark vector can also decay to $\bar{e} e$ at tree-level, with a partial width of

$$
\Gamma_{\chi \rightarrow \bar{e} e}=\frac{m_{\chi}}{24 \pi} \sqrt{1-\frac{4 m_{e}^{2}}{m_{\chi}^{2}}}\left\{\left[\left(g_{\chi e e}^{L}\right)^{2}+\left(g_{\chi e e}^{R}\right)^{2}\right]\left(1-\frac{m_{e}^{2}}{m_{\chi}^{2}}\right)+6 g_{\chi e e}^{L} g_{\chi e e}^{R} \frac{m_{e}^{2}}{m_{\chi}^{2}}\right\} .
$$

Figure 1 shows the VDM lifetime and branching ratios (BRs) as function of $m_{\chi}$. For $m_{\chi} \lesssim 30 \mathrm{keV}$, the dominant decay channel is into $\nu \bar{\nu}$, while for larger masses the $3 \gamma$ final state dominates up to the electron-positron threshold. For $m_{\chi}>2 m_{e}$, the $e^{+} e^{-}$channel is open and becomes the dominant decay mode, until $m_{\chi} \approx 25 \mathrm{MeV}$ from which the $3 \gamma$ final state, whose partial width grows quickly as $m_{\chi}^{9}$, dominates again. Note that BRs are independent of $g_{X}$, as well as $\theta_{R}$ in the absence of kinetic mixing. For most of the coupling range that is relevant for reproducing the DM relic density (see below) dark vectors with $m_{\chi}<2 m_{e}$ are typically stable on cosmological scale, with a VDM lifetime $\tau_{\chi}$ that largely exceeds the age of the Universe $\tau_{\mathrm{U}} \approx 4.3 \times 10^{17} \mathrm{~s}$ [48]. Conversely, dark vectors above the electron threshold are typically too short-lived to act as DM, thus we no longer consider this region henceforth. 


\section{The VDM relic density}

This section describes the production of VDM in the early universe. We assume that dark sector particles are initially absent from the thermal bath, that is $n_{\chi}=n_{E}=0$ at $T=T_{R}$ where $T_{R} \ll \Lambda$ is the reheating temperature. Because $E$ carries hypercharge, it will be vey quickly develop an equilibrium density from the scattering of hypercharge gauge boson provided $T_{R} \gg m_{E}$, which we assume here. Hence, $n_{E}=n_{\mathrm{eq}}$ for $T \lesssim T_{R}$. There are two distinct phases for the production of the $\chi_{\mu}$ relic density in the early Universe, depending on the size of its couplings to SM fields. If VDM interactions with the thermal bath are too slow, then the relic density will be produced mostly out-of-equilibrium, from a freeze-in mechanism where VDM particles are created by collision of thermal SM (and E) particles. Conversely, in the case that such interactions are faster than Hubble, $\chi_{\mu}$ will reach thermal equilibrium and its relic density will be set by thermal freeze-out.

There are two different types of processes that create VDM particles. First, $2 \rightarrow 2$ scattering processes with a photon, like $\ell_{1} \bar{\ell}_{2} \rightarrow \gamma \chi$ or $\ell_{1} \gamma \rightarrow \ell_{2} \chi$ with $\ell_{i}$ being either $e$ or $E$. Such processes, creating one VDM particle per collision, are allowed since $\chi_{\mu}$ is not stable. Double production from, for instance, $\ell_{1} \bar{\ell}_{2} \rightarrow \chi \chi$ is also possible. However, it is suppressed by a relative factor of $\sim g_{X}^{2} /(4 \pi \alpha)$, where $\alpha$ is the fine structure constant, and therefore negligible given the small values of $g_{X}$ considered.

Second, VDM can be produced by (in-equilibrium) decay of $E$ particles, $E \rightarrow \chi e$. This process is parametrically more efficient than $2 \rightarrow 2$ ones (suppressed by $\alpha$ ), since it requires one less power of equilibrium density in the initial state, and therefore largely dominates VDM production [13]. In contrast with VDM scenarios where production is possible through scattering [20], we show below that the decay channel allows to easily accomodate the observed dark matter abundance without conflicting with constraints from astrophysics and cosmology.

In the following, we first consider the regime of freeze-in VDM production from $E$ decays. We then discuss scattering contributions to the freeze-in mechanism, and show that those are neglegible, unless the mixing angle $\theta_{R}$ which controls the $E$ decay width, is very small (see figure 2). Finally, we consider the relativistic freeze-out limit. The resulting contour of $\Omega_{\chi} h^{2}=0.12$ is shown for illustration in figure 3 (black solid line) for $m_{E}=1 \mathrm{TeV}$ and $\theta_{R}=10^{-2}$.

\subsection{Freeze-in from heavy lepton decay}

The Boltzmann equation that determines the VDM number density $n_{\chi}$ produced from $E \rightarrow \chi e$ decay is

$$
\begin{aligned}
\dot{n}_{\chi}+3 H n_{\chi}= & \int d \Pi_{\chi} d \Pi_{E} d \Pi_{e}(2 \pi)^{4} \delta^{(4)}\left(p_{E}-p_{\chi}-p_{e}\right) \\
& \times\left[\left|\mathcal{M}_{E \rightarrow \chi e}\right|^{2} f_{E}\left(1-f_{e}\right)\left(1+f_{\chi}\right)-\left|\mathcal{M}_{\chi e \rightarrow E}\right|^{2} f_{\chi} f_{e}\left(1-f_{E}\right)\right],
\end{aligned}
$$

where $H \approx 1.66 \sqrt{g_{\rho}} T^{2} / m_{\mathrm{Pl}}$ is the Hubble rate (with $m_{\mathrm{Pl}}=1.22 \times 10^{19} \mathrm{GeV}$ the Planck mass and $g_{\rho}$ the number of degrees of freedom related to the energy density $\rho=\pi^{2} g_{\rho} T^{4} / 30$ ), $d \Pi_{i}=g_{i} \int d^{3} p_{i} /\left[(2 \pi)^{3} 2 E_{i}\right]$ and $f_{i}$ is the energy distribution of the particle $i$ in the plasma 
with $g_{i}$ spin degrees of freedom. The first term of the collision integral controls the production of $\chi_{\mu}$, and the second its depletion from inverse decay. The latter is only relevant in a regime of couplings where $\chi_{\mu}$ reaches equilibrium with the thermal bath.

In the freeze-in scenario, $f_{\chi} \simeq 0$ and the inverse decay is negligible. Further neglecting quantum statistical effects in the collision integral for the decay $E \rightarrow \chi e$, eq. (3.1) becomes

$$
\dot{n}_{\chi}+3 H n_{\chi}=n_{E}\left\langle\Gamma_{E \rightarrow \chi e}\right\rangle,
$$

where $\left\langle\Gamma_{E \rightarrow \chi e}\right\rangle=\Gamma_{E \rightarrow \chi e} m_{E} \int d^{3} p_{E}\left(f_{E} / E_{E}\right) / \int d^{3} p_{E} f_{E}$ is the thermal average of the decay rate $\Gamma_{E \rightarrow \chi e}$. The resulting comoving density $Y_{\chi} \equiv n_{\chi} / s$, with $s=2 \pi^{2} g_{s} T^{3} / 45$ the entropy density expressed in terms the number of degrees of freedom $g_{s}$, is approximately [13]

$$
Y_{\chi} \approx 4.3 \times 10^{-4} m_{E \mathrm{TeV}}^{-2}\left(\frac{\Gamma_{E \rightarrow \chi e}}{2.7 \times 10^{-5} \mathrm{eV}}\right)
$$

whose value is chosen such that the VDM relic abundance $\Omega_{\chi} h^{2} \approx 2.74 \times 10^{2} Y_{\chi} m_{\chi \mathrm{keV}}$ accommodates observations, $\Omega_{\mathrm{DM}} h^{2} \approx 0.12$ [48], for $\mathrm{keV}$-scale $\chi ; m_{E \mathrm{TeV}}$ denotes the $E$ mass in units of $\mathrm{TeV}$. In terms of the model parameters we obtain

$$
\Omega_{\chi} h^{2} \approx 0.12 \frac{m_{E \mathrm{TeV}}}{m_{\chi \mathrm{keV}}}\left(\frac{g_{X} \theta_{R}}{5.3 \times 10^{-17}}\right)^{2}
$$

The above derivation assumes that particles in the thermal bath have a Maxwell-Boltzmann energy distribution. One may question the validity of this approximation given that most of the DM is produced at temperatures around $T=m_{E} / 3$ from relativistic $E$ particles in association with an electron whose typical energy is $m_{E} / 2$, comparable to the temperature. When DM is produced from decays of thermal bath particles the effect of quantum statistics is typically important $[17,49]$, even more so if DM is produced in association with another thermal bath particle receiving little energy due to phase-space suppression [49]. Following ref. [49], we find that using Fermi-Dirac statistics for both the heavy lepton and the electron reduces the DM relic density by $\sim 20 \%$ relative to a classical treatment. This correction mostly originates from the Pauli-exclusion factor of the electron in eq. (3.1), which evaluates to $1-f_{e} \simeq 1-e^{-3 / 2} \approx 0.8$ at relevant temperatures.

Note that freeze-in production of the observed DM relic is only possible for $m_{\chi} \gtrsim 60 \mathrm{eV}$. Indeed, $Y_{\chi}$ cannot exceed the equilibrium value, which implies a lower bound on the VDM mass since $\Omega_{\chi} h^{2} \propto m_{\chi} Y_{\chi}$. Then, DM is rather produced by a freeze-out mechanism (see below).

Finally, VDM is also produced from out-of-equilibrium decay of $E$ particles after their density freezes out. This late-time contribution to the density of $\chi$ particles is given by the density of $E$ (and $\bar{E}$ ) particles at freeze-out $Y_{E}^{\text {fo }}$, multiplied by the $E \rightarrow \chi e$ branching ratio. $E$ and $\bar{E}$ are kept in chemical equilibrium with the thermal bath by hypercharge interactions and their freeze-out density is set by $E \bar{E} \rightarrow B B$ annihilation. The annihilation is $s$-wave with a thermally-averaged cross section of $\left\langle\sigma_{E \bar{E} \rightarrow B B} v\right\rangle \simeq g_{Y}^{4} /\left(8 \pi m_{E}^{2}\right)$. A standard freeze-out calculation [50] then yields $Y_{E}^{\mathrm{fo}} \simeq Y_{E}^{\mathrm{eq}}\left(T \approx m_{E} / 26\right) \approx 3 \times 10^{-12}$, with little 
dependence on the value of $m_{E}{ }^{2}$ In view of eq. (3.3), the fraction of VDM produced from out-of-equilibrium decay is therefore totally negligible relative to the freeze-in contribution.

\subsection{Subleading scattering contributions}

The discussion above is based on $\chi$ production from $E$ decay, and neglects sub-leading contributions from scattering. We provide here a more detailled analysis of the latter in order to establish the robustness of this approximation. While we use the full expressions for the scattering amplitudes in our numerical calculations, the corresponding DM yields can be estimated as follows. In the relativistic limit the $2 \rightarrow 2$ scattering rate scales as $n^{\mathrm{eq}}\langle\sigma v\rangle \sim \lambda^{2} T$ where $\lambda$ generically denotes the coupling constant of $\chi$ (including possible enhancement factor due to the longitudinal polarization of $\chi$ ) with the thermal bath and $n^{\text {eq }} \sim T^{3}$ is the equilibrium number density of the initial states. This reaction rate is to be compared with the Hubble rate which, during the radiation-dominated era, scales like $H \sim T^{2} / m_{\mathrm{Pl}}$. Hence, in this regime, DM production is more efficient the lower the temperature. Moreover, as soon as $T$ decreases below $m_{\mathrm{h}}$, the mass of the heaviest particle involved in the scattering, the reaction rate becomes exponentially suppressed and DM production quickly stops. Consequently, the final DM yield is approximately given by

$$
Y_{\chi}^{2 \rightarrow 2} \sim \frac{\lambda^{2}}{\bar{g}^{3 / 2}\left(m_{\mathrm{h}}\right)} \frac{m_{\mathrm{Pl}}}{m_{\mathrm{h}}}
$$

where $\bar{g}^{3 / 2} \equiv g_{s}^{2} / g_{*}^{1 / 2}$ with $g_{*}^{1 / 2}=\left(g_{s} / g_{\rho}^{1 / 2}\right)\left[1+1 / 3\left(d \log g_{s} / d \log T\right)\right]$. Similarly, the decay contribution is approximately $Y_{\chi}^{\text {decay }} \sim \kappa^{2} m_{\mathrm{Pl}} /\left[m_{E} \bar{g}^{3 / 2}\left(m_{E}\right)\right]$ where $\kappa$ is a generic decay constant $\left(\Gamma \propto \kappa^{2} m_{E}\right)$.

There are three types of $2 \rightarrow 2$ processes which involve a photon and either two $E$ particles, two electrons or one $E$ particle and an electron. We henceforth denote the total yield associated with these three contributions as $Y_{\chi}^{E E}, Y_{\chi}^{e e}$ and $Y_{\chi}^{E e}$, respectively.

In the absence of kinetic mixing, the effective couplings are $\lambda_{E E} \sim \sqrt{4 \pi \alpha} g_{\chi E E}^{V}, \lambda_{e e} \sim$ $\sqrt{4 \pi \alpha} g_{\chi e e}^{A}\left(m_{e} / m_{\chi}\right)$ and $\lambda_{E e} \sim \sqrt{4 \pi \alpha} g_{\chi E e}^{R}\left(m_{E} / m_{\chi}\right)$. The additional $m_{\ell} / m_{\chi} \gg 1$ factor in processes involving electrons stems from the fact the latter only couple to longitudinalypolarized $\chi$, which corresponds to the eaten Goldstone boson of the spontaneously broken $\mathrm{U}(1)_{X}$ gauge group, through the Yukawa operator in $\mathcal{L}_{\text {portal }}$. Note that only the axial part of $g_{\chi e e}$ is coupled to the longitudinal polatization, while the vector part is not enhanced by $m_{e} / m_{\chi}$. This is so because, in the vector-like limit, the global $\mathrm{U}(1)_{X}$ symmetry is preserved by the gauge boson mass term. Thus, the associated current is still conserved and the $1 / m_{\chi}^{2}$ term from the polarization sum does not contribute to the amplitude squared. Similarly, processes with two $E$ particles are controlled by $\mathrm{U}(1)_{X}$ gauge interactions, thus they are not enhanced by the longitudinal polarization factor. Furthermore, processes with at least one $E$ particle shut off rather early, near $T \sim m_{E}$, due to the heaviness of $E$, while processes with two electrons perdure until $T \sim m_{e} \ll m_{E}$ and are thus enhanced by a relative factor

\footnotetext{
${ }^{2}$ For $m_{E}$ close to the $\mathrm{TeV}$ scale, freeze-out typically occurs in the broken $\mathrm{SU}(2)_{L} \times \mathrm{U}(1)_{Y}$ phase where $B$ is no longer a proper mass eigenstate. However, $E$ has vector-like gauge interactions and the finiteness of the $Z$ boson mass only induces a small correction of $\sim m_{Z}^{2} / m_{E}^{2} \ll 1$ to the total annihilation cross section.
} 
of $m_{E} / m_{e} \times \bar{g}^{3 / 2}\left(m_{E}\right) / \bar{g}^{3 / 2}\left(m_{e}\right) \sim 10^{8} \times\left(m_{E} / \mathrm{TeV}\right)$, using $\bar{g}^{3 / 2}\left(m_{E}\right) / \bar{g}^{3 / 2}\left(m_{e}\right) \approx 40$. Hence, the yields from the different scattering processes are expected to scale as

$$
Y_{\chi}^{E e} \sim \frac{4 \pi \alpha g_{X}^{2} \theta_{R}^{2}}{\bar{g}^{3 / 2}\left(m_{E}\right)} \frac{m_{\mathrm{PI}} m_{E}}{m_{\chi}^{2}} \sim \theta_{R}^{2} \frac{m_{E}^{2}}{m_{\chi}^{2}} Y_{\chi}^{E E} \sim \frac{4}{\theta_{R}^{2}} \frac{\bar{g}^{3 / 2}\left(m_{e}\right)}{\bar{g}^{3 / 2}\left(m_{E}\right)} \frac{m_{E}}{m_{e}} Y_{\chi}^{e e}
$$

Taking $m_{E}=1 \mathrm{TeV}, Y_{\chi}^{e e}$ is thus always subdominant to $Y_{\chi}^{E e}$, as well as to $Y_{\chi}^{E E}$ whenever $\theta_{R} \lesssim 10^{-4}\left(m_{\chi \mathrm{keV}}\right)^{1 / 2}$, and $Y_{\chi}^{E e}$ dominates over $Y_{\chi}^{E E}$ for $\theta_{R} \gtrsim 10^{-9} m_{\chi \mathrm{keV}}$. In turn, the ratio $R$ of scattering to decay contributions to DM production

$$
R \equiv \frac{Y_{\chi}^{2 \rightarrow 2}}{Y_{\chi}^{\text {decay }}}
$$

is (neglecting the subleading contribution from two-electron processes)

$$
R(\epsilon=0) \sim 4 \pi \alpha\left(1+\frac{m_{\chi}^{2}}{\theta_{R}^{2} m_{E}^{2}}\right),
$$

where the effective coupling constant for decay $\kappa \sim g_{\chi E e}^{R}\left(m_{E} / m_{\chi}\right)$ is also enhanced because of the longitudinal polarization. This shows that DM production is dominated by decay unless $\theta_{R} \lesssim 3 \times 10^{-10} m_{\chi \mathrm{keV}}$, in which case DM is mostly produced by scattering processes of $E$ particles only.

Including a nonzero kinetic mixing $\epsilon=g_{X} g_{Y} /\left(6 \pi^{2}\right)$, the only significant change is in the yield from two-electron processes $Y_{\chi}^{e e}$. The $\chi$ coupling to electron pairs has now a vectorlike part whose contribution to the scattering cross section is dominated by transverse polarizations, as argued above. Consequently, the effective coupling is parametrically $\lambda_{e e} \sim$ $\sqrt{4 \pi \alpha} \sqrt{\left(g_{\chi e e}^{V}\right)^{2}+\left(g_{\chi e e}^{A}\right)^{2}\left(m_{e} / m_{\chi}\right)^{2}}$ and the scaling in eq. (3.6) is modified as

$$
Y_{\chi}^{e e}(\epsilon \neq 0) \sim\left(1+\frac{16 \alpha^{2}}{9 \pi^{2} \theta_{R}^{4}} \frac{m_{\chi}^{2}}{m_{e}^{2}}\right) Y_{\chi}^{e e}(\epsilon=0),
$$

where subleading term of $\mathcal{O}\left(\theta_{R}^{2}\right)$ has been neglected in $g_{\text {xee }}^{V}$ since typically $\theta_{R} \ll$ $\sqrt{4 \alpha /(3 \pi)} \approx 0.06$. Now, taking $m_{E}=1 \mathrm{TeV}, Y_{\chi}^{e e}$ always dominates over $Y_{\chi}^{E E}$, as well as $Y_{\chi}^{E e}$ whenever $\theta_{R} \lesssim 3 \times 10^{-8} m_{\chi \mathrm{keV}}$ and the scattering-to-decay ratio becomes, approximately,

$$
R(\epsilon \neq 0) \sim 4 \pi \alpha\left(1+\frac{16 \alpha^{2}}{9 \pi^{2} \theta_{R}^{2}} \frac{\bar{g}^{3 / 2}\left(m_{E}\right)}{\bar{g}^{3 / 2}\left(m_{e}\right)} \frac{m_{\chi}^{2}}{m_{e} m_{E}}\right) .
$$

Therefore, decay dominates DM production unless $\theta_{R} \lesssim 10^{-8} m_{\chi \mathrm{keV}}$, in which case the DM relic is set by scattering processes with two electrons.

The above estimates reproduce, up to $\mathcal{O}(1)$ factors, the full numerical calculation of the $R$ ratio whose results are presented in figure 2 for $\epsilon=0$ and $\epsilon=g_{X} g_{Y} /\left(6 \pi^{2}\right)$.

So far we have assumed that yields from $2 \rightarrow 2$ processes are dominated by temperatures comparable to the heaviest particle mass involved in the scattering. While this is generically true in vacuum, it is possible to get larger resonant contributions at higher temperatures once plasma effects are taken into account [20]. Photons in the plasma acquire a 


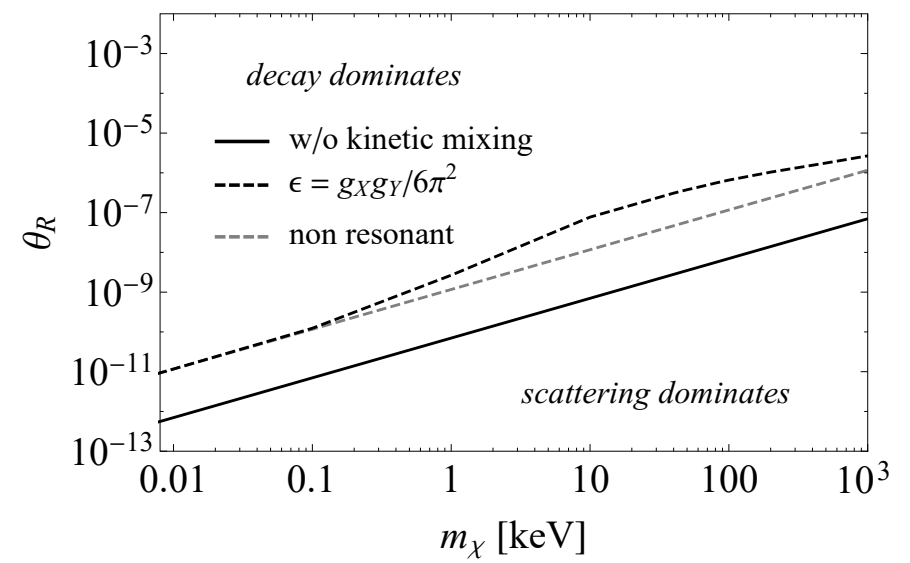

Figure 2. Regions where VDM production is dominated by decay $\left(R \equiv Y_{\chi}^{2 \rightarrow 2} / Y_{\chi}^{\text {decay }}<1\right)$ and $2 \rightarrow 2$ scattering processes $(R>1)$ in the $m_{\chi}-\theta_{R}$ plane, for cases without kinetic mixing (solid) and with $\epsilon=g_{X} g_{Y} /\left(6 \pi^{2}\right)$ (dashed), and assuming $m_{E}=1 \mathrm{TeV}$. The gray curve shows the scatteringto-decay ratio without the resonant enhancement from mixing with the photon in the plasma.

nonzero mass of $m_{\gamma}(T)=2 \alpha T^{2} / 3$ from interacting with thermal electrons [51]. As a result, the scattering yield is resonantlty enhanced, by mixing with the photon when its thermal mass equals that of the VDM. The VDM yield from such resonant photon conversion effect is particularly important for processes with two electrons when $m_{\chi}<2 m_{e}$ [20]. Note that resonant photon conversion is negligible for processes involving the heavier $E$ particles since the temperature $T_{r}$ at which resonance occurs is $\sim 8 m_{\chi} \ll m_{E}$ typically. Since it originates from mixing with the photon only the vector part of the electron coupling is involved. In the absence of kinetic mixing, the two-electron process contribution to the scattering yield is largely subdominant and its resonant enhancement is not relevant. It is however relevant in the case of nonzero kinetic mixing where $Y_{\chi}^{e e}$ dominates over $Y_{\chi}^{E E}$ and $Y_{\chi}^{E e}$. We calculated the resonant photon conversion contribution following ref. [20] and added it to the non resonant one evaluated without plasma effects. As shown in figure 2 the yield from photon conversion in the plasma typically exceeds the non resonant contribution by one to two orders of magnitude depending on the VDM mass. It is not significant for $m_{\chi} \lesssim 1 \mathrm{keV}$, which corresponds to $T_{r} \lesssim m_{e} / 3$, due to the Boltzmann-suppressed electron density in the plasma at these temperatures.

\subsection{The relativistic freeze-out limit}

For large enough values of $\Gamma_{E \rightarrow \chi e}$ the production of $\chi_{\mu}$ can be so efficient that the contribution from the inverse decay $\chi e \rightarrow E$ becomes relevant, eventually bringring VDM in equilibrium with the thermal bath if $\left\langle\Gamma_{E \rightarrow \chi e}\right\rangle \gg H$. In this case, the VDM abundance is rather set by a freeze-out mechanism.

For this to occur the rate of VDM interactions with the thermal bath must be faster than the Hubble rate before $E$ becomes non-relativistic, which corresponds to $n_{E} / n_{\chi} \times$ $\left\langle\Gamma_{E \rightarrow e \chi}\right\rangle / H \gtrsim 1$ at $T \approx m_{E} / 3$. Given the partial decay width of $E \rightarrow e \chi$ in eq. (2.12), the 
above thermalization condition implies a lower bound on the Yukawa portal coupling of ${ }^{3}$

$$
x_{E} \gtrsim 3.6 \times 10^{-7} \sqrt{m_{E T e V}},
$$

or, equivalently,

$$
g_{X} \gtrsim 2.5 \times 10^{-14} \frac{m_{\chi \mathrm{keV}}}{\sqrt{m_{E \mathrm{TeV}}}}\left(\frac{10^{-2}}{\theta_{R}}\right) .
$$

In this regime, the Boltzmann equation receives a contribution from the inverse decay process and reads ${ }^{4}$

$$
\dot{n}_{\chi}+3 H n_{\chi}=n_{E}\left\langle\Gamma_{E \rightarrow \chi e}\right\rangle\left(1-\frac{n_{\chi}}{n_{\chi}^{\mathrm{eq}}}\right),
$$

where $n_{\chi}^{\text {eq }}$ is the VDM equilibrium density. Chemical equilibrium with the SM is maintained as long as $E$ is abundant in the thermal bath, for $T \gg m_{E}$. When $T \lesssim m_{E}, E$ is no longer produced efficiently by thermal collisions and its density becomes exponentially suppressed. Eventually, this triggers the decoupling of $\chi_{\mu}$, while still relativistic, when the decay rate becomes slower than Hubble at $T=T_{f} \equiv m_{E} / x_{f}$ with $x_{f} \gtrsim 3$. Then, $Y_{\chi} \approx Y_{\chi}^{\mathrm{eq}}\left(T_{f} \gg m_{\chi}\right)$ is independent of the VDM mass and only mildly sensitive to the portal coupling $x_{E}$, through the value of $x_{f}$. This is in sharp contrast to the canonical non-relativistic freezeout scenario where the DM equilibrium density falls exponentially before decoupling, thus inducing a strong dependence on the DM coupling to the thermal bath.

Relativistic freeze-out occurs approximately when $n_{E} / n_{\chi}^{\mathrm{eq}}\left\langle\Gamma_{E \rightarrow \chi e}\right\rangle / H \lesssim 1$. Approximating the $E$ density as $n_{E} \simeq g_{E} e^{-m_{E} / T}\left[m_{E} /(2 \pi T)\right]^{3 / 2}$ and $\left\langle\Gamma_{E \rightarrow \chi e}\right\rangle \simeq \Gamma_{E \rightarrow \chi e}$, valid for $T \lesssim m_{E}$, yields

$$
x_{f} \simeq \log \left(\frac{4.7 g_{E} \Gamma_{E \rightarrow \chi e} m_{\mathrm{Pl}} g_{*}^{1 / 2}}{g_{\chi} m_{E}^{2} g_{s}}\right)+\frac{7}{2} \log \left[\log \left(\frac{4.7 g_{E} \Gamma_{E \rightarrow \chi e} m_{\mathrm{Pl}} g_{*}^{1 / 2}}{g_{\chi} m_{E}^{2} g_{s}}\right)\right],
$$

where $g_{E}=4$ and $g_{\chi}=3$ are spin degrees of freedom of $E$ (and $\bar{E}$ ) and $\chi$ particles. The variation with temperature of the numbers of degrees of freedom is typically negligible compared to the exponential falling of the $E$ number density, and $g_{s}$ and $g_{*}^{1 / 2}$ are assumed constant and evatuated at $T_{f}$ in eq. (3.14). The larger the decay width, the later VDM freezes out, as expected. For a leptonic portal coupling in the range $3.6 \times 10^{-7} \sqrt{m_{E T e V}}<$ $x_{E}<0.1$, one finds approximately $3.2 \lesssim x_{f} \lesssim 38-\log m_{E \mathrm{TeV}}$.

Consequently, $\Omega_{\chi}$ matches the observed abundance of DM only for a very narrow range of VDM masses, whose width reflects the mild sensitivity of $Y_{\chi}^{\text {eq }}$ to the value of $x_{f}$ (that is to the value of the VDM-to-SM coupling). Since $\chi$ is relativistic at freeze-out, $n_{\chi}^{\text {eq }} / T^{3}$ is constant and this dependence arises merely from the (possible) change in the number of entropy degrees of freedom during freeze-out. Assuming $\Omega_{\chi} h^{2} \approx 0.12$ yields

$$
m_{\chi} \approx 67 \mathrm{eV}\left[\frac{g_{s}\left(m_{E} / x_{f}\right)}{106.5}\right]
$$

\footnotetext{
${ }^{3}$ The reaction rate is understood here as being the sum of $E$ and $\bar{E}$ decays, namely $n_{E} \equiv g_{E} \int d^{3} p_{E} f_{E}$ with $g_{E}=4$.

${ }^{4}$ We assume that there is an efficient energy transfer between the SM and $\chi_{\mu}$ in order to write the reverse process contribution in terms of $n_{\chi} / n_{\chi}^{\text {eq }}$ in eq. (3.13). While this is not generically the case, it does however hold when $\chi_{\mu}$ is close to thermal equilibrium, which is the limit of interest here.
} 
where we use the value $g_{s} \approx 106.5$ for $m_{E}=1 \mathrm{TeV}$ and $x_{f}=3.2$. For $m_{\chi}$ above the value in eq. (3.15), VDM is overabundant, while for lighter masses $\chi$ cannot explain all the observed DM within the freeze-out regime.

Note that for relatively low $E$ mass values, a larger value of $x_{f}$ could imply a slightly different number of degrees of freedom . Consider for instance the extreme case of $x_{E}=0.1$, yielding a late freeze-out at $T_{f} \approx m_{E} / 38 \approx 26 \mathrm{GeV}$ for $m_{E}=1 \mathrm{TeV}$. At this temperature, the top quark, the Higgs and the weak gauge bosons have decoupled from the thermal bath and $g_{s}\left(T_{f}\right) \approx 89.6$. Then, a lower mass of $m_{\chi} \approx 56 \mathrm{eV}$ is required to accomodate the DM relic density. Conversely, for $m_{E} \gtrsim 6.3 \mathrm{TeV}$, freeze-out always occurs while all SM states are active and $g_{s}$ does not change. Hence, in this case, the relic density predicts a single VDM mass scale of $m_{\chi} \approx 67 \mathrm{eV}$, independently of VDM couplings.

At temperatures $T \ll m_{E}$, VDM could still be kept in thermal equilibrium through, now dominant, $2 \rightarrow 2$ scattering processes such as $\bar{e} e \rightarrow \chi \gamma$ and $\gamma e \rightarrow \chi e$ (and their inverse). However, due to the heaviness of $E$ and the smallness of the $\chi$ coupling to electrons, these two processes are found very inefficient and too slow to keep VDM in thermal equilibrium with the SM bath after the decoupling of $E$ particles.

\section{DM phenomenology and collider signatures}

Our VDM model has several possible experimental signatures. Those include DM absorption in direct detection searches, indirect astrophysical and cosmological probes from stellar cooling, $\chi \rightarrow 3 \gamma$ decays and cosmic microwave background (CMB). All of them are mostly sensitive to $g_{\chi e e}$. In addition, we consider also possible constraints from big-bang nucleosynthesis (BBN) and structure formation.

The VDM relic abundance fully determines the coupling $g_{\chi E e} \sim g_{X} \theta_{R}$ for fixed $m_{\chi}$ and $m_{E}$, see eq. (3.4). In absence of kinetic mixing, the VDM coupling to electrons scales as $g_{\chi e e}^{R} \sim g_{X} \theta_{R}^{2}$, thus all VDM experimental signatures are parametrically suppressed. Therefore, VDM can easily saturate the observed relic density while evading constraints from existing searches. However, in the presence of a nonvanishing kinetic mixing, the direct correlation between the relic density and experimental signatures is altered, since now $\epsilon$ contributes to $g_{\chi e e}$, but not to $g_{\chi E e}$. Assuming $\epsilon=g_{X} g_{Y} /\left(6 \pi^{2}\right), g_{\chi e e}$ is dominated by the kinetic mixing for $\theta_{R}<\sqrt{2 \alpha /(3 \pi)} \approx 4 \times 10^{-2}$. Therefore, for smaller $\theta_{R}$ values, saturation of the relic density requires larger values of $g_{X}$, resulting in an enhancement of the VDM signals in experiments.

We review below the searches relevant to our model and discuss the expected VDM signals in more details. The current constraints and future prospects on the VDM model are summarized in figure 3 in the $m_{\chi}-g_{X}$ plane for $m_{E}=1 \mathrm{TeV}, \theta_{R}=10^{-2}$ and assuming $\epsilon=0$ or $\epsilon=g_{X} g_{Y} / 6 \pi^{2}$.

\subsection{Direct detection}

Relic DM particles at the $\mathrm{MeV}$ scale or below are detectable through their interactions with atomic bound electrons. DM could either scatter or even be absorbed. In models where DM couples only feebly with the SM, scattering is typically very suppressed relative 

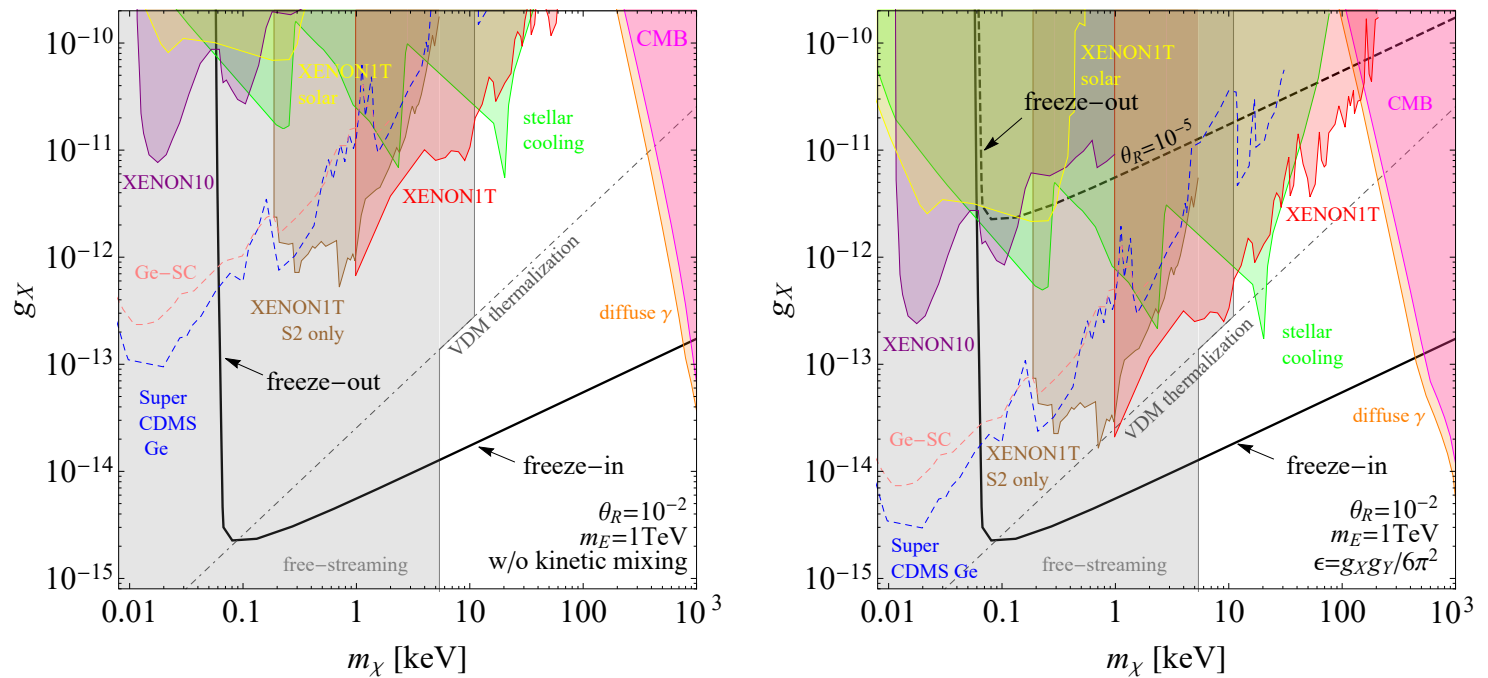

Figure 3. Constraints on VDM, $\chi_{\mu}$, in the $m_{\chi}-g_{X}$ plane for $m_{E}=1 \mathrm{TeV}, \theta_{R}=10^{-2}$ and $\epsilon=0$ (left) or $\epsilon=g_{X} g_{Y} / 6 \pi^{2}$ (right). The solid black line denotes the $\Omega_{\chi} h^{2}=0.12$ contour above (below) which VDM is over (under) abundant. Above the dot-dashed line VDM is in chemical equilibrium with the thermal bath and produced by freeze-out while relativistic; below, VDM is produced by freeze-in through $E \rightarrow \chi e$ decay. Shaded areas show regions excluded by searches of VDM absorption in direct detection experiments, including recast [25] from XENON10 [52] (purple) and XENON1T with electronic recoil data [53] (red) or ionisation signals (S2) only [54] (brown), solar emission (yellow), stellar cooling constraints [25], CMB anisotropies [55] (magenta), diffuse $\gamma$ ray background observations [56] following ref. [25] (orange) and structure formation (gray). Dashed lines denote projected sensitivities from future direct detection experiments like SuperCDMS, with Ge target [57] (blue), and using Ge semiconductor target [26] (pink).

to absorption as the cross section is quartic in small couplings and DM only deposites a fraction of its kinetic energy $m_{\chi} v_{\chi}^{2} / 2$ where $v_{\chi} \sim 10^{-3}$ is the average DM velocity in our galaxy. The VDM-electron scattering cross section [58] is typically extremely small ${ }^{5}$ $\sigma_{e} \simeq\left(g_{\chi e e}^{R}\right)^{4} /\left(8 \pi m_{\chi}^{2}\right) \sim \mathcal{O}\left(10^{-90} \mathrm{~cm}^{2}\right)$ for $\theta_{R}=10^{-2}$ and too many orders of magnitude below experimental sensitivities [54,59] to be observed.

Instead, absorption of VDM particles is more promissing. Not only DM deposits its entire mass energy when absorbed by a material but the associated cross section scales only quadratically in small couplings. The absorption signal is typically dominated by the vector part of the DM-electron coupling, contributions from the vector-axial part being suppressed by powers of the DM momentum-to-mass ratio. Here we adapt several existing bounds on absorption of canonical dark photon DM, namely scenarios where the dark photon couples to the SM only through kinetic mixing with the photon field strength, using the replacement rule $e \epsilon \rightarrow g_{\chi e e}^{V} \simeq g_{X} \theta_{R}^{2} / 2+e c_{W} \epsilon$.

\footnotetext{
${ }^{5}$ In the presence of kinetic mixing, the scattering amplitude receives extra contributions coming from the vector part of the electron coupling which is suppressed by a factor of $\mathcal{O}\left(m_{\chi}^{2} / m_{e}\right)$ due $\mathrm{U}(1)_{X}$ current conservation. The amplitude from virtual $E$ exchange is relatively suppressed by a factor of $\left(g_{\chi E e}^{R} / g_{\chi e e}^{R}\right)^{2} m_{\chi} m_{e} / m_{E}^{2} \approx 5 \times 10^{-16} \theta_{R}^{-2} m_{\chi \mathrm{keV}}^{2} m_{E \mathrm{TeV}}^{-4}$ and negligible.
} 
Of relevance are dark photon bounds extracted from recasts [25, 57] of XENON10 [52] and XENON100 [60] data, from XENON1T using electronic recoil data [53] or ionisation signals (S2) only [54], and from DAMIC [61] and SENSEI [59, 62, 63] experiments. XENON10, XENON100 and XENON1T are sensitive to absorption of DM whose mass is above their photoelectric threshold, namely $m_{\chi} \geq E_{\text {th }}=12.13 \mathrm{eV}$, while DAMIC and SENSEI have sensitivity to lower masses less relevant for the VDM parameter space under consideration. Direct detection experiments also serve as a target for dark photon emission from the Sun [64]. We use here the latest bound from a recast [65] of the XENON1T exclusion on solar emission [53]. Note that bremstrahlung contribution from the dark Higgs $\varphi$ is negligible here due to its heavy mass.

Resulting constraints and projections for our VDM scenario are presented in figure 3 on the $m_{\chi}-g_{X}$ plane for fixed $\theta_{R}=10^{-2}$. The shaded regions are excluded by DM searches by XENON10 (purple) and XENON1T (brown and red) and solar emission of new bosons by XENON1T (yellow). For sake of illustration of near future sensitivities on DM absorption in direct detection experiments, we show projections (dashed lines) of Ge semiconductor target with 1 kg-year exposure [26] (pink) and SuperCDMS Ge with $20 \mathrm{~kg}$-years exposure [57] (blue).

\subsection{Indirect detection}

Late $\chi \rightarrow 3 \gamma$ decays would contribute to the diffuse $\gamma$-ray background [20, 25]. Here, we follow ref. [25] and conservatively require that the sum of galactic and cosmological (extragalactic) VDM contributions to the diffuse $\gamma$-ray flux never exceeds the observed one [56]. For model parameters such that VDM production overshoots observations, we nevertheless assume that the VDM density at late time is $\Omega_{\chi} h^{2}=0.12$. In other words, we implicitly assume that the VDM density is subsequently diluted to the observed value by some unspecified mechanism. Conversely, for parameters yielding a VDM density below observations, we scale down the expected signal of ref. [25] by a factor of $\Omega_{\chi} h^{2} / 0.12$. The resulting exclusion is shown in figure 3 as an orange-shaded region. We note that indirect detection is a rather weak constraint on the region of parameter space consistent with the relic density, excluding only a small region below the electron-positron threshold, $m_{\chi} \lesssim 2 m_{e}$.

\subsection{Astrophysical and cosmological constraints}

We consider here possible constraints arising from VDM altering BBN and CMB physics, LSS formation and stellar evolution.

BBN. In constrast with usual WIMP scenarios, where DM is produced cold long before the onset of $\mathrm{BBN}$ at $T \sim \mathcal{O}(\mathrm{MeV})$, here VDM is produced hot and remains relativistic until after the formation of primordial nuclei. As a result, VDM would provide an additional contribution $\Delta N_{\text {eff }}^{\chi}$ to the effective number of neutrinos, $N_{\text {eff }}$. A too large $N_{\text {eff }}$ would hasten Hubble expansion compared to standard cosmology and thus modify light element abundances [66, 67]. 
Since VDM is not in equilibrium with the thermal bath at the time of BBN, its contribution to the effective number of neutrinos is [68]

$$
\Delta N_{\mathrm{eff}}^{\chi}=\frac{120}{7 \pi^{2}} \frac{\rho_{\chi}}{T_{\nu}^{4}}=\frac{4}{7} g_{\chi}\left(\frac{T_{\chi}}{T_{\nu}}\right)^{4}
$$

where $\rho_{\chi}$ is the VDM energy density, $T_{\chi}$ is the VDM temperature (in the case of kinetic equilibrium) and $T_{\nu} \sim \mathrm{MeV}$ is the temperature of neutrino decoupling. In the case that VDM ever was in thermal equilibrium with the SM, as in the freeze-out regime, its temperature after decoupling is fixed by entropic considerations. Once VDM decouples at $T=T_{f}=m_{E} / x_{f}$, its entropy and that of the SM are separately conserved. It then follows that the ratio $T_{\chi} / T$ is tied to the variation of the number of entropy degrees of freedom of the SM between $T$ and $T_{f}$ as $[68,69]$

$$
\frac{T_{\chi}}{T}=\left[\frac{g_{s}(T)}{g_{s}\left(T_{f}\right)}\right]^{1 / 3} .
$$

Taking $m_{E}=1 \mathrm{TeV}$, the lowest decoupling temperature is $T_{f} \approx 26 \mathrm{GeV}$ (corresponding to the case of large portal coupling, $x_{E}=0.1$ ). Then, combining eqs. (4.2) and (4.1), the VDM contribution to the effective number of neutrinos does not exceed

$$
\Delta N_{\mathrm{eff}}^{\chi} \lesssim 0.1
$$

Larger $E$ masses imply an earlier VDM decoupling, and a slightly smaller contribution to $N_{\text {eff. }}$.

In the freeze-in regime, however, VDM never reached equilibrium with the SM bath and eq. (4.2) does not apply. This case requires to solve an unintegrated Boltzmann equation in terms of the energy distribution of $f_{\chi}$ and evaluate the integral $\rho_{\chi}=\int_{m_{E}}^{\infty} E_{\chi} f_{\chi}\left(E_{\chi}\right)$. However, a rough estimate of the VDM energy density at $T \sim \mathcal{O}(\mathrm{MeV})$ shows that this is not necessary. Note that VDM particles are dominantly produced from $E$ decays at $T \sim m_{E}$ with a typical energy of $m_{E} / 2$ (up to a small spread due to the thermal kinetic energy of $E$ in the plasma). Since $\chi$ particles have very weak interactions with the SM bath (and among themselves), they cannot efficiently transfer energy to one another and their energy distribution merely redshifts with expansion. Therefore, at $T \sim \mathrm{MeV}$ we have approximately

$$
\rho_{\chi}(\mathrm{MeV}) \sim \frac{\mathrm{MeV}}{2} n_{\chi}(\mathrm{MeV}) \sim \frac{10^{-3}}{m_{\chi \mathrm{keV}}} \mathrm{MeV}^{4},
$$

where the second equality assumes $\Omega_{\chi} h^{2}=0.12$. Hence,

$$
\Delta N_{\mathrm{eff}}^{\chi} \sim 2 \times 10^{-3} m_{\chi \mathrm{keV}}^{-1} .
$$

which is at most $\Delta N_{\text {eff }}^{\chi} \lesssim 0.03$ given the lower bound $m_{\chi} \gtrsim 67 \mathrm{eV}$ in the freeze-in case.

The above VDM contributions, in eqs. (4.3) and (4.5), are to be compared to the value $N_{\text {eff }}=2.95 \pm 0.28(1 \sigma)$ [70] obtained from fitting helium and deuterium abundance observations within the standard BBN scenario. A value that is consistent with the SM 
expectation from neutrinos $N_{\text {eff }}^{\nu}=3.046$. Therefore, we conclude that VDM is not in tension with BBN.

Finally, note that while $E$ particles decouple relatively early, they do not decay right away to SM bath particles and $\chi$, due to the small couplings required to explain the DM relic abundance, most notably in the freeze-in regime. Summing the widths into $\chi e, Z e$ and $W \nu$ final state in eqs. (2.12) and (2.13), the $E$ lifetime is found to be

$$
\tau_{E} \simeq 2.3 \times 10^{-11} \mathrm{sec} \times \frac{m_{\chi \mathrm{keV}}}{m_{E \mathrm{TeV}}^{2}}\left(1+0.46 \frac{m_{\chi \mathrm{keV}}}{m_{E \mathrm{TeV}}}\right)^{-1}
$$

where $\Omega_{\chi} h^{2}=0.12$ was imposed, assuming freeze-in production of VDM and $\theta_{R}=10^{-2}$. Hence, $E$ disappears from the Universe long before BBN starts.

CMB. The $\chi \rightarrow 3 \gamma$ decays change the ionization history of the universe and leave imprints on the CMB in the form of observable temperature anisotropies, unless $\tau_{\chi \rightarrow 3 \gamma} \xi^{-1} \gtrsim$ $10^{25}$ sec [55] where $\xi \equiv \Omega_{\chi} / \Omega_{\mathrm{DM}}$ denotes the VDM fraction of the observed DM density. As for the constraint from diffuse $\gamma$-rays above, we implicitly assume some late dilution mechanism in the case of the overabundant $\chi$ production. The resulting exclusion, shown in figure 3 (magenta shaded region), is slightly weaker than the constraint from diffuse $\gamma$-ray observations, and only relevant for VDM masses close to the electron-positron threshold.

Structure formation. Once produced, DM particles propagate freely in the Universe, thus erasing matter density perturbations and, consequently, structures on scales smaller than the free-streaming length $\lambda_{\text {FS }}$. High-redshift observations, like the Lyman- $\alpha$ forest $[71$, 72], forbids strong suppression of the matter power spectrum below $\lambda_{\mathrm{FS}} \lesssim \mathcal{O}(0.1 \mathrm{Mpc})$ [41]. In order to estimate the resulting bound on the VDM parameter space, we follow ref. [16] and simply demand that the VDM free-streaming scale does not exceed 0.1 Mpc. $^{6}$

In the radiation-dominated era, the DM free-streaming scale is [75]

$$
\lambda_{\mathrm{FS}} \approx \frac{a_{\mathrm{NR}}}{H_{0} \sqrt{\Omega_{R}}}\left[0.62+\log \left(\frac{a_{\mathrm{eq}}}{a_{\mathrm{NR}}}\right)\right],
$$

where $a_{\text {eq }}=2.9 \times 10^{-4}$ is the scale factor of the Universe at the time of matter/radiation equality, $\Omega_{R}=9.3 \times 10^{-5}$ is the radiation density and $H_{0}=67.3 \mathrm{~km} \mathrm{~s}^{-1} \mathrm{Mpc}^{-1}$ is the Hubble's constant today. Numerical values of these cosmological parameters are taken from the results of the full-mission Planck measurements [76]. $a_{\mathrm{NR}}$ denotes the scale factor when the VDM became non-relativistic. The fact that momentum redshifts like $a^{-1}$ allows to relate to a different time, for instance the VDM decoupling time $t_{d}$, with

$$
a_{\mathrm{NR}}=\frac{\langle p\rangle_{d}}{\langle p\rangle_{\mathrm{NR}}} a_{d}
$$

where $\langle p\rangle$ is the averaged VDM momemtum, with $\langle p\rangle_{\mathrm{NR}}=m_{\chi}$. The scale factor $a_{d}$ is related to its value today by entrody dilution $a_{d}=a_{0}\left[g_{s}\left(T_{0}\right) / g_{s}\left(T_{d}\right)\right]^{1 / 3}\left(T_{0} / T_{d}\right)$, where $T=T_{0} \approx 2.3 \times 10^{-4} \mathrm{eV}$ and $a_{0}=1$, conventionally.

\footnotetext{
${ }^{6}$ Another approach, giving comparable estimates, consists in mapping analytically models of nonthermal DM to a warm DM (WDM) candidate and recast existent WDM analyses of the matter power spectrum $[73,74]$.
} 
In the freeze-out limit, VDM decouples at $T_{d}=T_{f}$ with a thermal distribution, giving $a_{\mathrm{NR}} \simeq 3.15 T_{0} / m_{\chi}\left[g_{s}\left(T_{0}\right) / g_{s}\left(T_{f}\right)\right]^{1 / 3}$ and

$$
\lambda_{F S}=\frac{0.86 \mathrm{Mpc}}{m_{\chi \mathrm{keV}}}\left(1+0.13 \log m_{\chi \mathrm{keV}}\right),
$$

taking $g_{s}\left(T_{0}\right) \approx 3.91$ and $g_{s}\left(T_{f}\right) \approx 106.5$. Forbidding VDM to stream freely on distances larger than $\sim 0.1 \mathrm{Mpc}$ implies a lower bound on the VDM mass of $m_{\chi} \gtrsim 11 \mathrm{keV}$. This scenario is thus excluded by structure formation since the correct relic density in the freeze-out limit requires $m_{\chi} \sim 60 \mathrm{eV}$.

The estimation of the free-streaming length differs in the freeze-in case since VDM never thermalized. Instead, most of it is produced at $T_{d} \sim m_{E} / 3$ with $\langle p\rangle_{d} \sim m_{E} / 2$. Consequently, $a_{\mathrm{NR}} \simeq 3 T_{0} /\left(2 m_{\chi}\right)\left[g_{s}\left(T_{0}\right) / g_{s}\left(T_{d}\right)\right]^{1 / 3}$ and

$$
\lambda_{F S}=\frac{0.45 \mathrm{Mpc}}{m_{\chi \mathrm{keV}}}\left(1+0.12 \log m_{\chi \mathrm{keV}}\right),
$$

yielding a lower bound on the VDM mass of

$$
m_{\chi} \gtrsim 5.4 \mathrm{keV}
$$

The above bounds are shown in figure 3 (gray shaded region) assuming all DM is in the form of VDM. In the region where the freeze-in production is overefficient the VDM relic density is implicitly assumed to be diluted at late times to the observed value. For the case that VDM is underabundant, a somewhat weaker constraint is expected [77].

Stellar cooling. Light particles of sub-MeV mass can be produced inside the hot and dense interior of stars. If weakly coupled to particles in the plasma, they will propagate without rescattering and eventually escape. Hence, the light particles produced will carry away energy and contribute an additional mechanism for stellar cooling, which is constrained by stellar modeling and observations [27-29]. Moreover, if the new particle mass is close to the plasma frequency in the stellar medium, emission happens resonantly which substantially enhances energy losses.

VDM particles produced within stellar cores follow from two processes: bremsstrahlung and inverse Compton scattering, both of which are sensitive to the VDM coupling to electron. Here we recast the bounds of refs. [25, 28, 29] obtained for dark photons using the Sun, Horizontal Branch (HB) stars and Red Giants (RGs), by requiring that VDM emission be at most $10 \%$ of the observed luminosity for the first two, and smaller than $10 / \mathrm{erg} / \mathrm{g} / \mathrm{s}$ for the last one. As for dark photons, the production of spin-1 particles is dominated by mixing with the photon in the stellar medium. This can be understood from the fact that, as far as only electrons are concerned which is the case in stars, it is always possible to redefine fields and move to a different basis where the vector coupling of the spin- 1 state to electrons is replaced by a kinetic mixing operator $\chi_{\mu \nu} F^{\mu \nu}$, where $F_{\mu \nu}$ denotes the QED field strength. As a result, the cooling mechanism is dominated by the vector part of the electron coupling. The resulting bounds are presented in figure 3 (green shaded region), where the three dips denote (from left to right) the best sensitivity from the Sun, HB stars and RGs. 


\subsection{Collider signatures}

For $m_{E}$ in the $\mathrm{TeV}$ range, the model studied above has also interesting collider signatures. While the DM itself cannot be detected at colliders, the heavy lepton $E$, which is an important ingredient allowing to reproduce the DM relic density, can be produced at the LHC or future colliders and searched for in multiple ways.

The dominant $E$ production mechanism is in pairs from EW interactions, namely through $q \bar{q}$ annihilating to off-shell $Z$ or $\gamma$ in the $s$-channel,

$$
q \bar{q} \rightarrow \gamma^{*} / Z^{*} \rightarrow E \bar{E}
$$

Using MAdGraPh 5 v2.7.3 [78], we estimate the $p p \rightarrow E E$ cross section at the LHC with $\sqrt{s}=13 \mathrm{TeV}$ to be $5.4 \times 10^{-2} \mathrm{fb}$ for $m_{E}=1 \mathrm{TeV}$. Single production of $E$ is also possible, yet at the expense of small mixing angle with the electron, leading to negligible cross sections at colliders.

Once produced, heavy leptons decay to $\chi e, Z e$ or $W \nu$, leading to different final states when $E$ decays promptly, as in the freeze-out regime. First, consider the case where $\Gamma_{E \rightarrow \chi e}$ dominates over the EW channels, leading to $\chi \chi e^{+} e^{-}$in the final state. This signal is very similar to that of chargino pairs decaying into dilepton plus missing energy [79, 80]. Currently, such searches bound the cross section for chargino pair production at the $\mathcal{O}(0.1) \mathrm{fb}$ level [81]. Hence, heavy leptons with $m_{E}=1 \mathrm{TeV}$ or higher are allowed in this case. Then, consider the opposite case where $E$ dominantely decays to EW channels, either $Z e$ or $W \nu$. This leads to clean signatures with multiple charged leptons in the final state, assuming the $Z$ and $W$ to decay leptonically. While a detailed analysis is beyond the scope of the this work, we note that current bounds from multilepton searches at the LHC does not reach yet the $\mathrm{TeV}$ scale mass region [82-84]. For instance, in the $Z Z e^{+} e^{-}$channel, taking at least one $Z$ to decay into $e^{+} e^{-}$or $\mu^{+} \mu^{-}$, the expected number of multilepton events is less than one for a data set of about $140 \mathrm{fb}^{-1}$ using the above estimate of the $p p \rightarrow E \bar{E}$ cross section at $\sqrt{s}=13 \mathrm{TeV}$. Therefore, we conclude that $m_{E}=1 \mathrm{TeV}$ is allowed by current experimental data.

For part of the parameter space, in particular when the VDM relic density is produced by freeze-in, $E$ can have finite decay length. For instance, imposing $\Omega_{\chi} h^{2}=0.12$ yields $c \tau_{E} \approx 1.5 \mathrm{~cm} /\left[\left(\theta_{R} / 10^{-2}\right)^{2}+2.2 m_{\chi \mathrm{keV}}^{-1}\right]$ for $m_{E}=1 \mathrm{TeV}$. Thus, LHC searches for long-lived charged particles [85-88] have some potential to probe TeV-scale heavy leptons that are either stable on collider scale or decay within the detector, notably for small values of the leptonic mixing angle.

Finally, precision electroweak observables can be modified in the presence of nonzero values of $\theta_{R}$ and/or the kinetic mixing parameter $\epsilon$. The latter induces a universal shift of the neutral current, whereas the former induces a breaking of lepton-flavor universality of the $Z$ couplings of $\mathcal{O}\left(\theta_{R}^{2}\right)$. Moreover, a nonzero kinetic mixing alters the relation between $m_{Z}$ and the SM parameters, hence contributing to the $\rho$ parameter, and the $Z$ decay width. However, in the limit of $m_{\chi} \ll m_{Z}$, the upper bound on $\epsilon$ is $\mathcal{O}\left(10^{-3}\right)[44,89,90]$, which is much larger that the typical loop-induced value considered here. Similarly, deviations from flavor universality in the lepton sector are excluded above the $10^{-3}$ level [91-93], 
corresponding to $\theta_{R} \lesssim 3 \times 10^{-2}$. We conclude that these bounds are too weak to constrain the region of parameter space relevant for DM.

\section{Conclusions}

In this work we considered models of decaying spin-1 DM $\chi$ associated with a spontaneously broken $\mathrm{U}(1)_{X}$ gauge symmetry. In constrast with previous models of this kind, the dominant interaction of DM with the SM sector is through a Yukawa portal where the dark-Higgs scalar connects a dark vector-like lepton $E$, charged under $\mathrm{U}(1)_{X}$, to the right-handed electron. In the $\mathrm{U}(1)_{X}$ broken phase, this portal induces a mass mixing between the dark lepton and the electron. There is no stabilizing symmetry for $\chi$. However, for small enough $\mathrm{U}(1)_{X}$ gauge coupling and leptonic mixing angle $\theta_{R}$, its decays are sufficiently suppressed to guarantee the stability of DM on cosmological scale. The DM relic abundance is set dominantly by $E \rightarrow \chi e$ decays in the early Universe. For small portal coupling values such that $\chi$ is never in thermal equilibrium with the SM bath, the DM abundance is produced by the freeze-in mechanism. Conversely, for coupling values larger than $\sim 10^{-7}, \chi$ reaches equilibrium before $E$ decouples and the DM abundance is instead set by a relativistic freeze-out mechanism. In the latter case, the comoving density of $\chi$ is almost independent of its mass and couplings, and the observed abundance is accomodated only for a specific mass of $m_{\chi} \sim 60 \mathrm{eV}$, with a mild dependence (few eVs) on the portal coupling through the effective number of degrees of freedom of the thermal bath at freezeout. However, this hot DM scenario is excluded by structure formation considerations. For higher $\chi$ masses, the correct relic abundance is obtained by the freeze-in mechanism. For $m_{\chi}>2 m_{e} \approx 1 \mathrm{MeV}, \chi$ decays too rapidly into electron pairs to form a valid DM candidate. We stress that successful DM phenomenology is achieved without resorting to ad-hod stabilizing symmetries, nor unnaturally small parameters since the model has an enhanced symmetry associated with $E$-number conservation in the limit of zero Yukawa portal coupling and gauge couplings always self-renormalize.

Then, we explored different experimental probes of such decaying spin-1 DM, including direct and indirect DM detection, energy losses in stars and the production of diffuse $\gamma$-rays. We also considered implications for BBN and CMB physics, and briefly outlined possible collider signatures in the case that the dark lepton lies around the $\mathrm{TeV}$ scale. In conclusion, we find that spin-1 DM in our model can explain the observed relic density while evading all existing constraints. Furthermore, this spin-1 decaying DM would most likely remain invisible also in the next round of DM detection and collider experiments, thus confering on such a DM candidate a stealth character. This feature is readily understood from the parametric relation between the relic density, which is set by $E \rightarrow \chi e$ decays whose rate are quadratic in $\theta_{R} \ll 1$, and the experimental signatures which rely on the DM coupling to electron pairs, suppressed here by higher powers of $\theta_{R}$.

We also discussed in our study the impact of having a nonzero kinetic mixing $\epsilon$ with the SM hypercharge. While it does not affect DM production in the early Universe, kinetic mixing does however breaks the latter correlation with DM searches by contributing to the (vector part of the) DM-electron coupling, $g_{\chi e e}^{V} \sim g_{X} \theta_{R}^{2} / 2+\epsilon e c_{W}$, which results in a stronger 
DM detection signals. Considering for illustration $\epsilon=g_{X} g_{Y} /\left(6 \pi^{2}\right)$, which is commensurate with the typical $E$ contribution at one-loop, the enhanced signals are still below current bounds for $\theta_{R} \sim \mathcal{O}\left(10^{-2}-10^{-4}\right)$. However, for smaller mixing angle, including values such that the relic density is dominantly set by scattering processes, only part of the observed DM can be explained within our model. All our results are summarized in figure 3 .

The model presented in this work admits various modifications, which can give different phenomenology. For instance, the new lepton can mix with $\mu$ and/or $\tau$, instead of the electron. While the relic density can be accomodated in a similar way, with the possibility to raise the DM mass up to the $2 \mu$ or $2 m_{\tau}$ threshold, this would however tear down hopes of direct DM detection, as these experiments rely on the DM coupling to electrons. Another possibility is mixing with the quark sector by introducing dark vector-like quarks, instead of leptons. Such models would bring about a distinct and rich phenomenology, most notably in terms of direct detection since DM could be at the $\mathrm{GeV}$ mass scale and couple to nucleons. Moreover, through CKM mixing effects dark sector contributions to flavor violating processes are unavoidable. Similarly, in leptophilic models, lepton-flavor violating processes such as $\mu \rightarrow e \gamma$ could be relevant, if vector-like leptons mix with more than one SM lepton flavor. However, such effects are expected be negligible due to the small $\mathrm{U}(1)_{X}$ gauge coupling needed for a valid DM candicate.

\section{Acknowledgments}

We thank Yi Fan Chen, Shlomit Tarem and Tomer Volansky for fruitful discussions and Geneviève Bélanger, Eric Kuflik and Yue Zhao for comments on the manuscript. TM is supported by the Israel Science Foundation (Grant No. 751/19). TM and YS are supported by the United States-Israel Binational Science Foundation (BSF) (NSF-BSF program Grant No. 2018683) and the Azrieli foundation. YS is Taub fellow (supported by the Taub Family Foundation).

Open Access. This article is distributed under the terms of the Creative Commons Attribution License (CC-BY 4.0), which permits any use, distribution and reproduction in any medium, provided the original author(s) and source are credited.

\section{References}

[1] M.W. Goodman and E. Witten, Detectability of certain dark matter candidates, Phys. Rev. D 31 (1985) 3059 [INSPIRE].

[2] LUX collaboration, Results from a search for dark matter in the complete LUX exposure, Phys. Rev. Lett. 118 (2017) 021303 [arXiv: 1608.07648] [INSPIRE].

[3] PandaX-II collaboration, Dark matter results from 54-ton-day exposure of PandaX-II experiment, Phys. Rev. Lett. 119 (2017) 181302 [arXiv:1708.06917] [INSPIRE].

[4] XENON collaboration, Dark matter search results from a one ton-year exposure of XENON1T, Phys. Rev. Lett. 121 (2018) 111302 [arXiv:1805.12562] [INSPIRE].

[5] ADMX collaboration, A search for invisible axion dark matter with the axion dark matter experiment, Phys. Rev. Lett. 120 (2018) 151301 [arXiv: 1804.05750] [INSPIRE]. 
[6] J.L. Ouellet et al., First results from ABRACADABRA-10 cm: a search for sub- $\mu \mathrm{eV}$ axion dark matter, Phys. Rev. Lett. 122 (2019) 121802 [arXiv:1810.12257] [INSPIRE].

[7] M. Battaglieri et al., U.S. cosmic visions: new ideas in dark matter 2017. Community report, in U.S. cosmic visions: new ideas in dark matter, FERMILAB-CONF-17-282-AE-PPD-T, (2017) [arXiv: 1707.04591] [INSPIRE].

[8] M. Garny, M. Sandora and M.S. Sloth, Planckian interacting massive particles as dark matter, Phys. Rev. Lett. 116 (2016) 101302 [arXiv:1511.03278] [INSPIRE].

[9] M. Garny, A. Palessandro, M. Sandora and M.S. Sloth, Theory and phenomenology of Planckian interacting massive particles as dark matter, JCAP 02 (2018) 027 [arXiv: 1709.09688] [INSPIRE].

[10] Y. Ema, K. Nakayama and Y. Tang, Production of purely gravitational dark matter, JHEP 09 (2018) 135 [arXiv: 1804.07471] [INSPIRE].

[11] M. Garny, A. Palessandro, M. Sandora and M.S. Sloth, Charged Planckian interacting dark matter, JCAP 01 (2019) 021 [arXiv:1810.01428] [INSPIRE].

[12] J. McDonald, Thermally generated gauge singlet scalars as selfinteracting dark matter, Phys. Rev. Lett. 88 (2002) 091304 [hep-ph/0106249] [INSPIRE].

[13] L.J. Hall, K. Jedamzik, J. March-Russell and S.M. West, Freeze-in production of FIMP dark matter, JHEP 03 (2010) 080 [arXiv: 0911.1120] [inSPIRE].

[14] N. Bernal, M. Heikinheimo, T. Tenkanen, K. Tuominen and V. Vaskonen, The dawn of FIMP dark matter: a review of models and constraints, Int. J. Mod. Phys. A 32 (2017) 1730023 [arXiv: 1706.07442] [INSPIRE].

[15] F. Elahi, C. Kolda and J. Unwin, Ultraviolet freeze-in, JHEP 03 (2015) 048 [arXiv: 1410.6157] [INSPIRE].

[16] A. Falkowski, E. Kuflik, N. Levi and T. Volansky, Light dark matter from leptogenesis, Phys. Rev. D 99 (2019) 015022 [arXiv: 1712.07652] [INSPIRE].

[17] S. Heeba, F. Kahlhoefer and P. Stöcker, Freeze-in production of decaying dark matter in five steps, JCAP 11 (2018) 048 [arXiv: 1809.04849] [INSPIRE].

[18] A. Fradette, M. Pospelov, J. Pradler and A. Ritz, Cosmological beam dump: constraints on dark scalars mixed with the Higgs boson, Phys. Rev. D 99 (2019) 075004 [arXiv: 1812.07585] [INSPIRE].

[19] M. Pospelov, A. Ritz and M.B. Voloshin, Bosonic super-WIMPs as keV-scale dark matter, Phys. Rev. D 78 (2008) 115012 [arXiv:0807.3279] [InSPIRE].

[20] J. Redondo and M. Postma, Massive hidden photons as lukewarm dark matter, JCAP 02 (2009) 005 [arXiv: 0811.0326] [INSPIRE].

[21] J.D. Bjorken, R. Essig, P. Schuster and N. Toro, New fixed-target experiments to search for dark gauge forces, Phys. Rev. D 80 (2009) 075018 [arXiv:0906.0580] [InSPIRE].

[22] L.B. Okun, Limits of electrodynamics: paraphotons?, Sov. Phys. JETP 56 (1982) $502[$ [Zh. Eksp. Teor. Fiz. 83 (1982) 892] [INSPIRE].

[23] P. Galison and A. Manohar, Two Z's or not two Z's?, Phys. Lett. B 136 (1984) 279 [INSPIRE].

[24] B. Holdom, Two U(1)'s and epsilon charge shifts, Phys. Lett. B 166 (1986) 196 [InSPIRE]. 
[25] H. An, M. Pospelov, J. Pradler and A. Ritz, Direct detection constraints on dark photon dark matter, Phys. Lett. B 747 (2015) 331 [arXiv:1412.8378] [INSPIRE].

[26] Y. Hochberg, T. Lin and K.M. Zurek, Absorption of light dark matter in semiconductors, Phys. Rev. D 95 (2017) 023013 [arXiv:1608.01994] [INSPIRE].

[27] J. Redondo, Helioscope bounds on hidden sector photons, JCAP 07 (2008) 008 [arXiv: 0801.1527] [INSPIRE].

[28] H. An, M. Pospelov and J. Pradler, New stellar constraints on dark photons, Phys. Lett. B 725 (2013) 190 [arXiv:1302.3884] [INSPIRE].

[29] J. Redondo and G. Raffelt, Solar constraints on hidden photons re-visited, JCAP 08 (2013) 034 [arXiv: 1305.2920] [INSPIRE].

[30] S.D. McDermott and S.J. Witte, Cosmological evolution of light dark photon dark matter, Phys. Rev. D 101 (2020) 063030 [arXiv:1911.05086] [InSPIRE].

[31] A.E. Nelson and J. Scholtz, Dark light, dark matter and the misalignment mechanism, Phys. Rev. D 84 (2011) 103501 [arXiv:1105.2812] [INSPIRE].

[32] P.W. Graham, J. Mardon and S. Rajendran, Vector dark matter from inflationary fluctuations, Phys. Rev. D 93 (2016) 103520 [arXiv:1504.02102] [InSPIRE].

[33] P. Agrawal, N. Kitajima, M. Reece, T. Sekiguchi and F. Takahashi, Relic abundance of dark photon dark matter, Phys. Lett. B 801 (2020) 135136 [arXiv:1810.07188] [INSPIRE].

[34] R.T. Co, A. Pierce, Z. Zhang and Y. Zhao, Dark photon dark matter produced by axion oscillations, Phys. Rev. D 99 (2019) 075002 [arXiv:1810.07196] [INSPIRE].

[35] J.A. Dror, K. Harigaya and V. Narayan, Parametric resonance production of ultralight vector dark matter, Phys. Rev. D 99 (2019) 035036 [arXiv: 1810.07195] [InSPIRE].

[36] M. Bastero-Gil, J. Santiago, L. Ubaldi and R. Vega-Morales, Vector dark matter production at the end of inflation, JCAP 04 (2019) 015 [arXiv: 1810.07208] [INSPIRE].

[37] G. Choi, T.T. Yanagida and N. Yokozaki, Dark photon dark matter in the minimal B $-L$ model, JHEP 01 (2021) 057 [arXiv:2008.12180] [INSPIRE].

[38] R.T. D'Agnolo and J.T. Ruderman, Light dark matter from forbidden channels, Phys. Rev. Lett. 115 (2015) 061301 [arXiv:1505.07107] [INSPIRE].

[39] S. Colombi, S. Dodelson and L.M. Widrow, Large scale structure tests of warm dark matter, Astrophys. J. 458 (1996) 1 [astro-ph/9505029] [INSPIRE].

[40] P. Bode, J.P. Ostriker and N. Turok, Halo formation in warm dark matter models, Astrophys. J. 556 (2001) 93 [astro-ph/0010389] [INSPIRE].

[41] M. Viel, G.D. Becker, J.S. Bolton and M.G. Haehnelt, Warm dark matter as a solution to the small scale crisis: new constraints from high redshift Lyman- $\alpha$ forest data, Phys. Rev. D 88 (2013) 043502 [arXiv: 1306.2314] [INSPIRE].

[42] G. Arcadi, A. Djouadi and M. Raidal, Dark matter through the Higgs portal, Phys. Rept. 842 (2020) 1 [arXiv: 1903.03616] [INSPIRE].

[43] S. Cassel, D.M. Ghilencea and G.G. Ross, Electroweak and dark matter constraints on a $Z^{\prime}$ in models with a hidden valley, Nucl. Phys. B 827 (2010) 256 [arXiv:0903.1118] [INSPIRE]. 
[44] J.M. Cline, G. Dupuis, Z. Liu and W. Xue, The windows for kinetically mixed $Z^{\prime}$-mediated dark matter and the galactic center gamma ray excess, JHEP 08 (2014) 131

[arXiv: 1405.7691] [INSPIRE].

[45] C.-N. Yang, Selection rules for the dematerialization of a particle into two photons, Phys. Rev. 77 (1950) 242 [INSPIRE].

[46] W. Heisenberg and H. Euler, Consequences of Dirac's theory of positrons, Z. Phys. 98 (1936) 714 [physics/0605038] [inSPIRE].

[47] S.D. McDermott, H.H. Patel and H. Ramani, Dark photon decay beyond the Euler-Heisenberg limit, Phys. Rev. D 97 (2018) 073005 [arXiv:1705.00619] [InSPIRE].

[48] Planck collaboration, Planck 2018 results. VI. Cosmological parameters, Astron. Astrophys. 641 (2020) A6 [arXiv:1807.06209] [INSPIRE].

[49] G. Bélanger, F. Boudjema, A. Goudelis, A. Pukhov and B. Zaldivar, MicrOMEGAs5.0: freeze-in, Comput. Phys. Commun. 231 (2018) 173 [arXiv:1801.03509] [InSPIRE].

[50] P. Gondolo and G. Gelmini, Cosmic abundances of stable particles: improved analysis, Nucl. Phys. B 360 (1991) 145 [INSPIRE].

[51] H.A. Weldon, Covariant calculations at finite temperature: the relativistic plasma, Phys. Rev. D 26 (1982) 1394 [inSPIRE].

[52] XENON10 collaboration, A search for light dark matter in XENON10 data, Phys. Rev. Lett. 107 (2011) 051301 [Erratum ibid. 110 (2013) 249901] [arXiv: 1104.3088] [INSPIRE].

[53] XENON collaboration, Excess electronic recoil events in XENON1T, Phys. Rev. D 102 (2020) 072004 [arXiv: 2006.09721] [INSPIRE].

[54] XENON collaboration, Light dark matter search with ionization signals in XENON1T, Phys. Rev. Lett. 123 (2019) 251801 [arXiv:1907.11485] [INSPIRE].

[55] V. Poulin, J. Lesgourgues and P.D. Serpico, Cosmological constraints on exotic injection of electromagnetic energy, JCAP 03 (2017) 043 [arXiv: 1610.10051] [INSPIRE].

[56] H. Yuksel and M.D. Kistler, Circumscribing late dark matter decays model independently, Phys. Rev. D 78 (2008) 023502 [arXiv:0711.2906] [INSPIRE].

[57] I.M. Bloch, R. Essig, K. Tobioka, T. Volansky and T.-T. Yu, Searching for dark absorption with direct detection experiments, JHEP 06 (2017) 087 [arXiv: 1608.02123] [INSPIRE].

[58] R. Essig, J. Mardon and T. Volansky, Direct detection of sub-GeV dark matter, Phys. Rev. D 85 (2012) 076007 [arXiv: 1108.5383] [INSPIRE].

[59] SENSEI collaboration, SENSEI: direct-detection results on sub-GeV dark matter from a new skipper-CCD, Phys. Rev. Lett. 125 (2020) 171802 [arXiv: 2004.11378] [INSPIRE].

[60] XENON100 collaboration, First axion results from the XENON100 experiment, Phys. Rev. D 90 (2014) 062009 [Erratum ibid. 95 (2017) 029904] [arXiv: 1404.1455] [INSPIRE].

[61] DAMIC collaboration, First direct-detection constraints on eV-scale hidden-photon dark matter with DAMIC at SNOLAB, Phys. Rev. Lett. 118 (2017) 141803 [arXiv:1611.03066] [INSPIRE].

[62] SENSEI collaboration, Single-electron and single-photon sensitivity with a silicon skipper CCD, Phys. Rev. Lett. 119 (2017) 131802 [arXiv:1706.00028] [InSPIRE]. 
[63] SENSEI collaboration, SENSEI: direct-detection constraints on sub-GeV dark matter from a shallow underground run using a prototype skipper-CCD, Phys. Rev. Lett. 122 (2019) 161801 [arXiv: 1901.10478] [INSPIRE].

[64] H. An, M. Pospelov and J. Pradler, Dark matter detectors as dark photon helioscopes, Phys. Rev. Lett. 111 (2013) 041302 [arXiv: 1304.3461] [InSPIRE].

[65] H. An, M. Pospelov, J. Pradler and A. Ritz, New limits on dark photons from solar emission and keV scale dark matter, Phys. Rev. D 102 (2020) 115022 [arXiv:2006.13929] [INSPIRE].

[66] V.F. Shvartsman, Density of relict particles with zero rest mass in the universe, Pisma Zh. Eksp. Teor. Fiz. 9 (1969) 315 [JETP Lett. 9 (1969) 184] [INSPIRE].

[67] G. Steigman, D.N. Schramm and J.E. Gunn, Cosmological limits to the number of massive leptons, Phys. Lett. B 66 (1977) 202 [INSPIRE].

[68] S. Sarkar, Big bang nucleosynthesis and physics beyond the Standard Model, Rept. Prog. Phys. 59 (1996) 1493 [hep-ph/9602260] [INSPIRE].

[69] K.A. Olive, D.N. Schramm and G. Steigman, Limits on new superweakly interacting particles from primordial nucleosynthesis, Nucl. Phys. B 180 (1981) 497 [INSPIRE].

[70] R.H. Cyburt, B.D. Fields, K.A. Olive and T.-H. Yeh, Big bang nucleosynthesis: 2015, Rev. Mod. Phys. 88 (2016) 015004 [arXiv: 1505.01076] [InSPIRE].

[71] R.A.C. Croft et al., Towards a precise measurement of matter clustering: Lyman- $\alpha$ forest data at redshifts 2-4, Astrophys. J. 581 (2002) 20 [astro-ph/0012324] [INSPIRE].

[72] U. Seljak, A. Slosar and P. McDonald, Cosmological parameters from combining the Lyman- $\alpha$ forest with CMB, galaxy clustering and SN constraints, JCAP 10 (2006) 014 [astro-ph/0604335] [INSPIRE].

[73] A. Kamada and K. Yanagi, Constraining FIMP from the structure formation of the universe: analytic mapping from $m_{W D M}$, JCAP 11 (2019) 029 [arXiv: 1907.04558] [INSPIRE].

[74] R. Huo, Matter power spectrum of light freeze-in dark matter: with or without self-interaction, Phys. Lett. B 802 (2020) 135251 [arXiv:1907.02454] [INSPIRE].

[75] E.W. Kolb and M.S. Turner, The early universe, Front. Phys. 69 (1990) 1 [InSPIRE].

[76] Planck collaboration, Planck 2015 results. XIII. Cosmological parameters, Astron. Astrophys. 594 (2016) A13 [arXiv:1502.01589] [INSPIRE].

[77] A. Boyarsky, J. Lesgourgues, O. Ruchayskiy and M. Viel, Lyman- $\alpha$ constraints on warm and on warm-plus-cold dark matter models, JCAP 05 (2009) 012 [arXiv:0812.0010] [INSPIRE].

[78] J. Alwall et al., The automated computation of tree-level and next-to-leading order differential cross sections, and their matching to parton shower simulations, JHEP 07 (2014) 079 [arXiv: 1405.0301] [INSPIRE].

[79] CMS collaboration, Searches for electroweak production of charginos, neutralinos, and sleptons decaying to leptons and $W, Z$, and Higgs bosons in pp collisions at $8 \mathrm{TeV}$, Eur. Phys. J. C $\mathbf{7 4}$ (2014) 3036 [arXiv:1405.7570] [INSPIRE].

[80] CMS collaboration, Search for supersymmetry in final states with two oppositely charged same-flavor leptons and missing transverse momentum in proton-proton collisions at $\sqrt{s}=13 \mathrm{TeV}$, arXiv:2012.08600 [INSPIRE].

[81] ATLAS collaboration, Search for electroweak production of charginos and sleptons decaying into final states with two leptons and missing transverse momentum in $\sqrt{s}=13$ TeV pp collisions using the ATLAS detector, Eur. Phys. J. C 80 (2020) 123 [arXiv:1908.08215] [INSPIRE]. 
[82] CMS collaboration, Search for physics beyond the Standard Model in multilepton final states in proton-proton collisions at $\sqrt{s}=13 \mathrm{TeV}$, JHEP 03 (2020) 051 [arXiv:1911.04968] [INSPIRE].

[83] CMS collaboration, Search for vector-like leptons in multilepton final states in proton-proton collisions at $\sqrt{s}=13 \mathrm{TeV}$, Phys. Rev. D 100 (2019) 052003 [arXiv: 1905.10853] [INSPIRE].

[84] ATLAS collaboration, Search for supersymmetry in events with four or more leptons in $\sqrt{s}=13$ TeV pp collisions with ATLAS, Phys. Rev. D 98 (2018) 032009 [arXiv:1804.03602] [INSPIRE].

[85] ATLAS collaboration, Search for heavy charged long-lived particles in the ATLAS detector in $36.1 \mathrm{fb}^{-1}$ of proton-proton collision data at $\sqrt{\mathrm{s}}=13 \mathrm{TeV}$, Phys. Rev. D 99 (2019) 092007 [arXiv: 1902.01636] [INSPIRE].

[86] CMS collaboration, Search for long-lived charged particles in proton-proton collisions at $\sqrt{s}=13 \mathrm{TeV}$, Phys. Rev. D 94 (2016) 112004 [arXiv: 1609.08382] [InSPIRE].

[87] ATLAS collaboration, Search for heavy long-lived charged R-hadrons with the ATLAS detector in $3.2 \mathrm{fb}^{-1}$ of proton-proton collision data at $\sqrt{s}=13 \mathrm{TeV}$, Phys. Lett. $B \mathbf{7 6 0}$ (2016) 647 [arXiv: 1606. 05129] [INSPIRE].

[88] ATLAS collaboration, Searches for heavy long-lived charged particles with the ATLAS detector in proton-proton collisions at $\sqrt{s}=8 \mathrm{TeV}$, JHEP 01 (2015) 068 [arXiv:1411.6795] [INSPIRE].

[89] A. Hook, E. Izaguirre and J.G. Wacker, Model independent bounds on kinetic mixing, Adv. High Energy Phys. 2011 (2011) 859762 [arXiv: 1006.0973] [INSPIRE].

[90] D. Curtin, R. Essig, S. Gori and J. Shelton, Illuminating dark photons with high-energy colliders, JHEP 02 (2015) 157 [arXiv:1412.0018] [INSPIRE].

[91] A. Efrati, A. Falkowski and Y. Soreq, Electroweak constraints on flavorful effective theories, JHEP 07 (2015) 018 [arXiv: 1503.07872] [INSPIRE].

[92] W. Altmannshofer, S. Gori, M. Pospelov and I. Yavin, Quark flavor transitions in $L_{\mu}-L_{\tau}$ models, Phys. Rev. D 89 (2014) 095033 [arXiv: 1403.1269] [INSPIRE].

[93] A. Falkowski and D. Straub, Flavourful SMEFT likelihood for Higgs and electroweak data, JHEP 04 (2020) 066 [arXiv: 1911.07866] [INSPIRE]. 